\title{
Whispering Gallery Waves in a Neighborhood of a Higher Order Zero of the Curvature of the Boundary
}

\author{
By \\ Gen Nakamura*, Yoshihiro Shibata** and Kazumi Tanuma***
}

\section{$\S \mathbb{1}$. Introduction}

Let $\Omega \subset \boldsymbol{R}^{2}$ be a domain with a smooth boundary $\Gamma$. Suppose $\Omega$ is a media where some wave propagates with a speed, say 1 for simplicity. When $\Omega$ is strictly convex, there is a high frequency wave well known by the name of whispering gallery waves which propagates along $\Gamma$. Mathematically, it can be described as an asymptotic solution $u$ to the reduced wave equauion

$$
\Delta u+\omega^{2} u=0 \quad \text { in } \Omega
$$

with the Dirichlet boundary condition

$$
\left.u\right|_{\Gamma}=0
$$

whose energy is concentrated near $\Gamma$.

Here $\omega \gg 1$ is the frequency of the wave, and $u$ satisfies (1.1) asymptotically as $\omega \rightarrow \infty$. By using the boundary layer method, Babich and Kirpichnikova [1] constructed an asymptotic solution $u$ which describes the whispering gallery wave.

However, if we merely assume that $\Omega$ is convex, their asymptotic solution is ineffective in a neighborhood of a point $P \in \Gamma$ at which the curvature $K_{0}$ of $\Gamma$ is zero, i.e. one of its first order derivatives blows up at $P$.

On the other hand, Popov [4] considered the case $K_{0}(P)=0, K_{0}^{\prime}(P) \neq 0$. He constructed an asymptotic solution $u$ to (1.1) and (1.2) in a relatively open neighborhood $G \subset \bar{\Omega}$ of $P$ with the following property. Namely, the dominant term of $u$ converges in the $L^{2}$ sense to that of whispering gallery wave constructed in $[1]$ as $\omega \rightarrow \infty$ in a relatively open neighborhood $G_{+} \subset G$ of $\Gamma_{+}=$

Communicated by S. Matsuura, January 26, 1988.

* Department of Mathematics, Josai University, Saitama 350-02, Japan.

** Institute of Mathematics, University of Tsukuba, Ibaraki 305, Japan.

*** Department of Mathematics, School of Science and Engineering, Waseda University, Tokyo 160, Japan. 
$\left\{Q \in \Gamma \cap G ; K_{0}(Q)>0\right\}$. (In $\S 2$ we will give the precise definition of $G_{+}$) Moreover, he showed that, by appropriate coordinate transformation and scaling transformation, the dominant term of $u$ is determined by a certain Dirichlet boundary value problem for a certain type of Schrödinger equation with an unbounded potential, and constructed its formal solution on the image of $G_{+}$under the composite mapping of these transformations.

In this paper, we have extended the results of Popov under the condition

$$
\Gamma_{+} \neq \varnothing, \quad K_{0}^{(\jmath)}(P)=0 \quad(j<\kappa), \quad K_{0}^{(\kappa)}(P) \neq 0
$$

for some positive integer $\kappa$. Moreover, we have improved his result in the following two points. (1) The dominant term $\phi$ (cf. (2.12) below) of the asymptotic solution $u$ to (1.1) and (1.2) is smooth up to the boundary of $G_{+}$. (2) $\psi$ converges to the dominant term $\phi_{0}$ (cf. (2.9) below) of the asymptotic solution of the whispering gallary wave constructed in [1] with respect to a higher regularity norm as $\omega \rightarrow \infty$ on $G_{+}$.

Although we have restricted our study to improving Popov's result, our analysis is a starting point for constructing a local paramatrix which describes the gliding wave generated by an incident wave grazing at an inflection point at the boundary.

Recently, we have noticed that Babich and Smyshlyaev [2] obtained the same result for the special case $\kappa=1$. However, their method is quite different from ours.

The rest of this paper is organized as follows. After some preparations, we state the results of [1], [4] and our results in $\S 2$. $\S 3$ and $\S 4$ are devoted to the proof of our main theorem.

Acknowledgement. We appreciate the referee for several valuable comments on revising this paper.

\section{$\S 2$. Some Preparations and Results}

Let $s$ be the arclength of $\Gamma$ measured from a fixed point $P_{0}$ of $\Gamma$, and $\Gamma$ is described by $\vec{\tau}=\vec{\tau}(s)$. Let $\vec{n}(s)$ be the unit normal vector at $\vec{\tau}(s)$ pointing inward to $\Omega$ and $q$ be the length measured along $\vec{n}(s)$. Then we can take $(s, q)$ as a coordinate in a relatively open neighborhood $G \subset \bar{\Omega}$ of $\Gamma$. Thus each point $M=M(x, y) \in G$ with a cartesian coordinate $(x, y)$ is represented in the form

$$
M=M(s, q)=\vec{\tau}(s)+q \vec{n}(s) .
$$

When the wave propagates with speed $c(x, y)$, the corresponding Dirichlet boundary value problem becomes 


$$
\left\{\begin{array}{l}
\Delta u+\omega^{2} c^{-2}(x, y) u=0 \quad \text { in } \Omega \\
\left.u\right|_{\Gamma}=0 .
\end{array}\right.
$$

Now by taking the center of curvature in $\Omega$, let $K_{0}(s)$ be the curvature of $\Gamma$ at $s$. Then we define the effective curvature $K(s)$ by

$$
K(s)=K_{0}(s)+\left.c^{-1}(s, 0) \cdot \partial_{q} c(s, q)\right|_{q=0} .
$$

Clearly, $K(s)=K_{0}(s)$ if the speed $c(s, q)$ of the wave is constant. For the wave with non constant speed, we have to replace the condition (1.3) by

$$
\begin{array}{ll}
\Gamma_{+}=\{s \in \Gamma \cap G ; K(s)>0\} \neq \varnothing, \quad & K^{(\jmath)}(0)=0 \quad(j<\kappa), \\
& K^{(\kappa)}(0) \neq 0 .
\end{array}
$$

Here we have taken $P=P_{0}=(0,0)$ and identified $Q \in \Gamma$ with $s$. Hence $K(s)=$ $\delta s^{\kappa}+O\left(s^{\kappa+1}\right)(s \rightarrow+0)$ for some $\delta>0$. Moreover we define $G_{+}$by $G_{+}=\{(s, q) \in G$; $K(s)>0\}$. In terms of the coordinate $(s, q)$, (2.1) becomes

$$
\left\{\begin{array}{c}
\left(1-q K_{0}(s)\right)^{-1}\left[\partial_{s}\left\{\left(1-q K_{0}(s)\right)^{-1} \partial_{s}\right\}+\partial_{q}\left\{\left(1-q K_{0}(s)\right) \partial_{q}\right\}\right] U \\
+\omega^{2} c^{-2}(s, q) U=0 \quad \text { in } G \\
U_{q=0}^{\prime}=0 .
\end{array}\right.
$$

Hereafter, we mainly use (2.4) instead of (2.1) and we construct an asymptotic solution $U(=u)$ of $(2.4)$ with the properties stated in $\S 1$.

Let

$$
v(x)=\int_{0}^{\infty} \cos \left(t^{3} / 3+x t\right) d t \quad(x \in \boldsymbol{R})
$$

be one of the Airy functions which is rapidly decreasing as $x \rightarrow+\infty$ together with all of its derivatives and has the zeros only on the negative real axis. Now let $-\nu<0$ be a zero of $v(x)$. Then corresponding to this zero, there is an asymptotic solution $U$ to $(2.4)$ on $G_{+}$which describes the whispering gallery wave. More precisely we have the following. (see pp. $37 \sim 47$ in [1])

Theorem 2.1. For any sufficiently small $\varepsilon>0$, there is an asymptotic solution $U$ of $(2.4)$ in $G_{+}(\varepsilon)=\{(s, q) \in G ; K(s)>\varepsilon\}$ with the following properties.

1. $U$ admits the following asymptotic expansion as $\omega \rightarrow \infty$ :

$$
U(s, q) \sim \exp \left\{i \omega \int_{s_{0}}^{s} c^{-1}(s, 0) d s+i \omega^{1 / 3} h(s)\right\} \cdot \sum_{n=0}^{\infty} \omega^{-n / 3} U_{n}(s, q)
$$

where

$$
\begin{aligned}
& h(s)=-\nu \int_{s_{0}}^{s} K^{2 / 3}(s)[2 c(s, 0)]^{-1 / 3} d s, \\
& U_{0}=[2 K(s) c(s, 0)]^{1 / 6} \cdot v\left(\omega^{2 / 3} q \eta(s)-\nu\right),
\end{aligned}
$$




$$
\eta(s)=[2 K(s)]^{1 / 3} \cdot c^{-2 / 3}(s, 0),
$$

and $s_{0} \in \Gamma$ with $K\left(s_{0}\right)>\varepsilon$.

2. $U_{n}(n \geqq 1)$ can be obtained successively by solving a certain recursive equation. In particular, $U_{1}$ is given by

where

$$
\begin{aligned}
U_{1}= & {\left[( - i / 2 ) c ^ { 1 / 2 } ( s , 0 ) ( 2 K ( s ) ) ^ { - 1 / 2 } \left\{(2 / 3)(2 K(s))^{-1} K^{\prime}(s)\right.\right.} \\
& \left.\left.-(2 / 3) c^{-1}(s, 0) \partial_{s} c(s, 0)\right\} y^{2}+A_{1}(s)\right] v(y-\nu)
\end{aligned}
$$

$$
y=\omega^{2 / 3} q \eta(s)
$$

and $A_{1}(s)$ is a $C^{\infty}$ function in $s$.

Remark. Each $U_{n}(n \geqq 2)$ is a linear combination of $v(y-\nu)$ and $v^{\prime}(y-\nu)$ whose coefficients are $C^{\infty}$ function in $s$. By the asymptotic behavior of $v(x)$ as $x \rightarrow+\infty$ and the definition of $-\nu$, we can easily see that the asymptotic solution $U(s, q)$ decays exponentially as $\omega \rightarrow \infty$ if $q>0$ and $U(s, q)$ satisfies the Dirichlet boundary condition. Thus this asymptotic solution corresponds to the whispering gallery wave. From (2.6), the asymptotic solution $U(s, q)$ is no longer valid as $s \rightarrow 0$.

Our next aim is to construct an asymptotic solution $U$ to (2.4) in $G$ which still has a meaning at $s=0$ and behaves like the whispering gallery wave in $G_{+}$. As we had already assumed at the beginning of this section, note that

$$
K(s)=\delta s^{\kappa}+O\left(s^{\kappa+1}\right) \quad(s \rightarrow+0)
$$

for some $\delta>0$ and positive integer $\kappa$. Introduce the scaling transformation

$$
\left\{\begin{array}{l}
t=s \omega^{1 /(2 \kappa+3)}\left[\delta^{2} c^{-1}\right]^{1 /(2 \kappa+3)} \\
x=q \omega^{(\kappa+2) /(2 \kappa+3)}\left[\delta c^{-\kappa-2}\right]^{1 /(2 \kappa+3)}
\end{array}\right.
$$

where $c=c(0,0)$, and $U_{0}$ is the one given by (2.6). Then

$$
\exp \left(i \omega^{1 / 3} h(s)\right) U_{0}=\phi_{0}(t, x)\left(1+O\left(\omega^{-1 /(2 \kappa+3)}\right)\right) \quad(\omega \rightarrow \infty)
$$

where

$$
\begin{gathered}
\left.\phi_{0}(t, x)=D 2^{1 / 6} t^{\kappa / 6} \exp \{-i \nu(3 / 2 \kappa+3)) 2^{-1 / 3} t^{(2 \kappa+3) / 3}\right\} v\left(2^{1 / 3} t^{\kappa / 3} x-\nu\right) \\
D=C^{(\kappa+1) /(4 \kappa+6)} \delta^{1 /(4 \kappa+6)} \omega^{-\kappa /(12 \kappa+18)} \exp \left(-i \omega^{1 / 3} \nu \int_{s_{0}}^{0} K^{2 / 3}(s)(2 c(s, 0))^{-1 / 3} d s\right) .
\end{gathered}
$$

The following theorem is a simple extension of Popov's result (cf. [4]).

Theorem 2.2. There is an asymptotic solution $U$ to (2.4) in a relatively open neighborhood $G \subset \bar{\Omega}$ of $(s, q)=(0,0)$ with the following properties.

(1) $U$ admits the following asymptotic expansion as $\omega \rightarrow \infty$ : 


$$
U=\exp \left(i \omega \int_{s_{0}}^{s} c^{-1}(s, 0) d s\right) V(s, q)
$$

$$
V(s, q) \sim \sum_{n=0}^{\infty} \omega^{-n /(2 \kappa+3)} V_{n}(t, x) .
$$

(2) $V_{0}(t, x)=\psi(t, x) \in L^{2}\left(\boldsymbol{R} \times \boldsymbol{R}^{+}\right)$is uniquely determined by the following conditions :

$$
\begin{cases}L \psi=\left(i \partial_{t}+(1 / 2) \partial_{x}^{2}-x t^{\hbar}\right) \psi=0 & (t \in \boldsymbol{R}, x \geqq 0), \\ \left.\phi\right|_{x=0}=0 \quad(t \in \boldsymbol{R}), & \\ \left\|\phi(t, \cdot)-\psi_{0}(t, \cdot)\right\|_{L^{2}\left(R^{+}\right)} \longrightarrow 0 & (t \rightarrow+\infty) .\end{cases}
$$

Here $\phi_{0}(t, x)$ is the one given by (2.10).

(3) (2.12) admits the following formal solution $\phi_{\infty}(t, x)$ as $t \rightarrow+\infty$.

$$
\psi_{\infty}(t, x)=D 2^{1 / 6} t^{\kappa / 6} \exp \left\{-i \nu(3 /(2 \kappa+3)) 2^{-1 / 3} t^{(2 \kappa+3) / 3}\right\} \sum_{n=0}^{\infty} t^{-(2 \kappa+3) n / 3} \Phi_{n}(X)
$$

where

$$
\begin{gathered}
X=2^{1 / 3} t^{\kappa / 3} x, \quad \Phi_{0}(X)=v(X-\nu) . \\
\Phi_{n}(X)=P_{2 n}(X) v(X-\nu)+Q_{2 n-1}(X) v^{\prime}(X-\nu) \quad(n \geqq 1)
\end{gathered}
$$

for some polynomials $P_{2 n}(X)$ and $Q_{2 n-1}(X)\left(Q_{2 n-1}(0)=0\right)$ of respective orders $2 n$ and $2 n-1$.

(4) For each nonnegative integer $N$, let $\phi_{N}$ be the truncated sum of $\phi_{\infty}(t, x)$ up to $n=N$ and $f_{N}(t, x)=L \phi_{N}$. Then for any nonnegative integer $k, l, m, n$ satisfying

$$
\begin{gathered}
(4 \kappa+6) N+5-\kappa \geqq 6 k+4 \kappa m+2 \kappa n, \\
\operatorname{Sup}_{x \geqq 0}\left|t^{k} x^{\imath} \partial_{t}^{m} \partial_{x}^{n} f_{N}(t, x)\right| \longrightarrow 0 \quad(t \rightarrow+\infty)
\end{gathered}
$$

holds.

Remarks. 1. When $\kappa=1$, Theorem 2.2 is exactly the same as Popov's result (cf. [4]). Since the proof of Theorem 2.2 can be done in the same way as that of Popov's result (cf. [4]), we omit the proof.

2. Since $s>0$ in $G_{+}, t \rightarrow+\infty$ as $\omega \rightarrow \infty$ in $G_{+}$. Noting this and (2.9) (2.12), the dominant term of the asymptotic solution $U$ given in Theorem 2.2 approaches that of the whispering gallery wave with respect to the $L^{2}$ norm as $\omega \rightarrow \infty$ in $G_{+}$.

3. $\phi_{0}(t, x)$ is the first term in the formal expansion (2.13) of $\phi$.

Let $\chi(t) \in C^{\infty}\left(\overline{\boldsymbol{R}}^{+}\right)$satisfy

$$
\chi(t)=0 \quad(0 \leqq t \leqq 1), \quad \chi(t)=1 \quad(t \geqq 2) .
$$

Then we can easily see that, for any nonnegative integer $k, l, m, n, j$, 


$$
\left|t^{k} x^{l} \partial_{t}^{m} \partial_{x}^{n}\left\{t^{-(2 \kappa+3) j / 3} \Phi_{\jmath}(X) \chi\left(\varepsilon_{j} t\right)\right\}\right| \leqq C_{l m n j} t^{k-\kappa l / 3-m+\kappa n / 3-(2 \kappa+3) j / 3}
$$

where $C_{l m n_{j}}>0$ is a constant which does not depend on $1>\varepsilon_{j}>0$. Now take $\varepsilon_{j}$ $(j=0,1, \cdots)$ such that

$$
C_{l m n} \varepsilon_{j} \leqq 2^{-j} \quad \text { for any } l, m, n, j \quad(l+m+n \leqq j)
$$

and define $\psi_{N}(t, x), \tilde{\psi}_{N}(t, x)(N=0,1, \cdots), \tilde{\psi}(t, x)$ by the following equations

$$
\begin{aligned}
& \phi_{N}(t, x)=D 2^{1 / 6} t^{\kappa / 6} \exp \left\{-i \nu(3 /(2 \kappa+3)) 2^{-1 / 3} t^{(2 \tilde{\kappa}+3) / 3}\right\} \sum_{j=0}^{N} t^{-(2 \kappa+3) j / 3} \Phi_{\jmath}(X) \\
& \tilde{\phi}_{N}(t, x)=D 2^{1 / 6} t^{\kappa / 6} \exp \left\{-i \nu(3 /(2 \kappa+3)) 2^{-1 / 3} t^{(2 \kappa+3) / 3}\right\} \sum_{j=0}^{N} t^{-(2, i+3) \jmath / 3} \Phi_{\jmath}(X) \chi\left(\varepsilon_{j} t\right) \\
& \tilde{\psi}(t, x)=D 2^{1 / 6} t^{\kappa / 6} \exp \left\{-i \nu(3 /(2 \kappa+3)) 2^{-1 / 3} t^{(2 \kappa+3) / 3}\right\} \sum_{j=0}^{\infty} t^{-(2, x+3) \jmath / 3} \Phi_{\jmath}(X) \chi\left(\varepsilon_{j} t\right) .
\end{aligned}
$$

From (2.16) and (2.17) we can easily see

$$
\operatorname{Sup}_{x \geqq 0}\left|t^{k} x^{l} \partial_{t}^{m} \partial_{x}^{n} \sum_{j=N+1}^{\infty}\left\{t^{-(2 \kappa+3) j / 3} \Phi_{J}(X) \chi\left(\varepsilon_{j} t\right)\right\}\right| \leqq t^{k-\kappa l / 3-m+\kappa n / 3-(2 \kappa+3) N / 3+1}
$$

for nonnegative integers $k, l, m, n(l+m+n \leqq N)$. Moreover we have

$$
\operatorname{Sup}_{x \geq 0}\left|t^{k} x^{l} \partial_{t}^{m} \partial_{x}^{n}\left\{t^{-(2 \kappa+3) j / 3} \Phi_{j}(X)\left(\chi(t)-\chi\left(\varepsilon_{j} t\right)\right)\right\}\right| \longrightarrow 0(t \rightarrow+\infty)
$$

for nonnegative integers $k, l, m, n$.

Our next result gives a more detailed information about the asymptotic behavior of the first term $\phi$ of the asymptotic solution given in Theorem 2.2.

Theorem 2.3. (Main result). There exists

$$
\psi(t, x) \in L^{2}\left(\boldsymbol{R} \times \mathbb{R}^{+}\right) \cap C^{\infty}\left(\overline{\mathbb{R}}^{+} \times \overline{\mathbb{R}}^{+}\right) \cap\left(\bigcap_{\delta>0} \mathcal{S}\left(\overline{\boldsymbol{R}}^{+} \times[\delta, \infty)\right)\right)
$$

with the following properties.

(1) $\psi$ is a solution to

$$
\left\{\begin{array}{l}
L \phi=i \partial_{t} \psi+(1 / 2) \partial_{x}^{2} \psi-x t^{\kappa} \psi=0 \quad(t \in \mathbb{R}, x \geqq 0) . \\
\left.\phi\right|_{x=0}=0 .
\end{array}\right.
$$

(2) For each fixed $t \geqq 0, \phi(t, \cdot) \in \mathcal{S}\left(\overline{\boldsymbol{R}}^{+}\right)$.

(3) Given a nonnegative integer $N$, we have

$$
\operatorname{Sup}_{x \geqq 0}\left|t^{k} x^{l} \partial_{t}^{m} \partial_{x}^{n}\left(\psi-\phi_{N}\right)(t, x)\right| \longrightarrow 0 \quad(t \rightarrow+\infty)
$$

for any nonnegative integer $k, l, m, n$ with $3 k+2 \kappa m+\kappa n \leqq(2 \kappa+3) N+2 \kappa+2$.

(4) Moreover

$$
\operatorname{Sup}_{x \geq 0}\left|t^{k} x^{l} \partial_{t}^{m} \partial_{x}^{n}(\psi-\tilde{\psi})(t, x)\right| \longrightarrow 0 \quad(t \rightarrow+\infty) .
$$


for any nonnegative integer $k, l, m, n$.

\section{$\S 3$. Proof of Main Theorem (Part 1)}

Let $N$ be a nonnegative integer and $h(t, x) \in \bigcap_{\delta>0} \mathcal{S}\left(\overline{\boldsymbol{R}}^{+} \times[\delta, \infty)\right)$ satisfy $h_{(}(+, 0)$ $=0$ and the estimate

$$
\operatorname{Sup}_{x \geq 0}\left|t^{k} x^{l} \partial_{t}^{m} \partial_{x}^{n} h(t, x)\right| \longrightarrow 0 \quad(t \rightarrow+\infty)
$$

for any nonnegative integer $k, l, m, n$ with

$$
k+l+n+n \leqq N .
$$

Consider the following unique solution $w(t, x) \in L^{2}\left(\boldsymbol{R} \times \boldsymbol{R}^{+}\right)$to

$$
\left\{\begin{array}{l}
L w=h(t, x) \quad(i \in \boldsymbol{R}, x \geqq 0) \\
\left.w\right|_{x=0}=0 \\
w(t, \cdot) \longrightarrow 0 \quad(t \rightarrow+\infty) \text { in } L^{2}\left(\boldsymbol{R}^{+}\right) .
\end{array}\right.
$$

Here we claim the following. Claim: There is a function $\rho(N)$ such that $\rho^{\prime}(N)$ $\rightarrow \infty$ as $N \rightarrow \infty$ and

$$
\operatorname{Sup}_{x \geq 0}\left|t^{k} x^{l} \partial_{t}^{m} \partial_{x}^{n} w(t, x)\right| \longrightarrow 0 \quad(t \rightarrow+\infty)
$$

for any nonnegative integer $k, l, m, n$ with

$$
k+l+m+n \leqq \rho(N) .
$$

We postpone the proof of this claim for a while and show that the properties (3) (4) of Theorem 2.3 follows from the claim.

As for (4), let $h=-L(\tilde{\psi})$ and $\chi(t) \in C^{\infty}\left(\overline{\boldsymbol{R}}^{+}\right)$be the function already given by (2.15). If we decompose $h$ into the form

$$
h=-L\left(\chi(t) \phi_{N}\right)-L\left(\tilde{\psi}_{N}-\chi(t) \phi_{N}\right)-L\left(\tilde{\psi}-\tilde{\psi}_{N}\right)
$$

and remained the estimates (2.14), (2.19), (2.18), we have (3.1) for any nonnegative integer $k, l, m, n$. Then (4) immediately follows from the claim.

As for (3), fix nonnegative integers $N, l$ and let $k, m, n$ be nonnegative integers which satisfy $3 k+2 \kappa m+\kappa n \leqq(2 \kappa+3) N+2 \kappa+2$. Take nonnegative integers $N^{\prime} \geqq N$ and $M$ so that

and

$$
\rho(M) \geqq l+(2 \kappa+3) N+2 \kappa+2
$$

$$
\operatorname{Sup}_{x \geq 0}\left|t^{k} x^{l} \partial_{t}^{m} \partial_{x}^{n} L\left(\chi(t) \psi_{N^{\prime}}\right)\right| \longrightarrow 0 \quad(t \rightarrow+\infty)
$$

for any nonnegative integer $k, l, m, n$ with

$$
k+l+m+n \leqq M \text {. }
$$


Since $w=\psi-\chi(t) \phi_{N^{\prime}}$ is the solution to (3.2) with $h=-L\left(\chi(t) \phi_{N^{\prime}}\right)$ and

$$
\operatorname{Sup}_{x \geq 0}\left|t^{k} x^{l} \partial_{t}^{m} \partial_{x}^{n}\left(\phi_{N^{\prime}}-\psi_{N}\right)\right| \longrightarrow 0 \quad(t \rightarrow+\infty)
$$

for any nonnegative integer $k, l, m, n$ with

$$
3 k+2 \kappa m+\kappa n \leqq(2 \kappa+3) N+2 \kappa+2,
$$

(3) immediately follows from the claim.

Now let us give the outline of the proof of the claim. By Duhamel's principle, $w(t, x)$ is given by

$$
w(t, x)=(1 / i) \int_{t}^{\infty} u(t, x ; T) d T
$$

if $u(t, x ; T)$ is the strong $L^{2}$ solution to the following problem:

$$
\left\{\begin{array}{l}
i \partial_{t} u+(1 / 2) \partial_{x}^{2} u-x t^{\kappa} u=0 \quad(t \in \mathbb{R}, x \geqq 0) \\
\left.u\right|_{x=0}=0 \\
\left.u\right|_{t=T}=h(T, x)
\end{array}\right.
$$

Hence it is enough to show the existence, regularity and decay of $u(t, x ; T)$ $(0 \leqq t \leqq T, x \geqq 0)$.

In this section, we prove the existence and the uniqueness of the strong $L^{2}$ solution $u(t, x ; T)$ to (3.5) in each finite time interval $\left[T^{*}, T\right]$. Then in the next section, we extend $u(t, x ; T)$ to $t \geqq 0$ and prove its regularity and decay by using certain energy inequalities.

Let $T^{*}, T$ satisfy $0<T^{*}<T$ and fix them. Applying Cauchy's method, we will construct the unique solution to the problem

$$
\left\{\begin{array}{l}
i \partial_{t} u+(1 / 2) \partial_{x}^{2} u-x t^{\kappa} u=0 \quad\left(T^{*}<t<T, x>0\right) \\
\left.u\right|_{x=0}=0 \quad\left(T^{*} \leqq t \leqq T\right) \\
\left.u\right|_{t=T}=h(T, x) \quad(x \geqq 0) .
\end{array}\right.
$$

For this purpose, let

$$
\Delta: T^{*}=t_{m}<t_{m-1}<\cdots<t_{1}<t_{0}=T
$$

be a partition of $\left[T^{*}, T\right]$ and define $u_{\Delta}(t, x)$ by

$$
u_{\Delta}(t, x)=u_{\Delta}^{(k)}(t, x) \quad\left(k=0,1, \cdots, m-1 ; t_{k+1} \leqq t \leqq t_{k}\right)
$$

where each $u_{\Delta}^{(k)}(t, x)$ is the unique solution to the problem

$$
\left\{\begin{array}{l}
i \partial_{t} u_{\Delta}^{(k)}+(1 / 2) \partial_{x}^{2} u_{\Delta}^{(k)}-x t_{k}{ }^{k} u_{\Delta}^{(k)}=0 \quad\left(t_{k+1}<t<t_{k}, x>0\right) \\
\left.u_{\Delta}^{(k)}\right|_{x=0}=0 \quad\left(t_{k+1} \leqq t \leqq t_{k}\right. \\
\left.u_{\Delta}^{(k)}\right|_{t=t_{k}}=u_{\Delta}^{(k-1)}\left(t_{k}, x\right) \quad(x \geqq 0)
\end{array}\right.
$$


such that

$$
u_{\Delta}^{(-1)}\left(t_{0}, x\right)=h(T, x) .
$$

Now let $z_{n}$ be the $n$-th negative zero of the Airy function $v(x)$ and set

$$
\begin{gathered}
\lambda_{n}\left(t_{k}\right)=-z_{n} 2^{-1 / 3} t_{k}{ }^{{ }^{h / 3}} \\
\psi_{n}\left(t_{k}, x\right)=-2^{1 / 6} t_{k}{ }^{\kappa / 6} v(z) /\left(\left.v^{\prime}(z)\right|_{x=0}\right)
\end{gathered}
$$

where $z=2^{1 / 3} t_{k}{ }^{-2 \kappa / 3}\left(t_{k}{ }^{k} x-\lambda_{n}\left(t_{k}\right)\right)$. Then according to Titchmarsh ([5] pp. 90 92), for each $k(0 \leqq k \leqq m-1),\left\{\psi_{n}\left(t_{k}, x\right)\right\}_{n=0}^{\infty}$ are eigenfunctions of

$$
H\left(t_{k}\right)=(-1 / 2) \partial_{x}{ }^{2}+x t_{k}{ }^{k}
$$

with eigenvalues $\left\{\lambda_{n}\left(t_{k}\right)\right\}_{n=0}^{\infty}$ satisfying the Dirichlet boundary condition and form an orthonormal system of $L^{2}\left(\boldsymbol{R}^{+}\right)$.

Next we define several norms and function spaces associated to these norms. Let $H_{0}^{1}\left(\boldsymbol{R}^{+}\right)$be the completion of $C_{0}^{\infty}\left(\boldsymbol{R}^{+}\right)$with respect to the Sobolev norm $\|\cdot\|_{w_{2}^{1}\left(R^{+}\right)}$and $K$ be Sobolev's constant such that

$$
\left|u^{\prime}(0)\right| \leqq K\left(\|u\|_{\left.L^{2\left(R^{+}\right.}\right)}+\left\|u^{\prime}\right\|_{L^{2}\left(R^{+}\right)}+\left\|u^{\prime \prime}\right\|_{L^{2}\left(R^{+}\right)}\right) .
$$

Definition 3.1. For $u(x)=\sum_{n=0}^{\infty} u_{n} \psi_{n}\left(x, t_{k}\right) \in \mathcal{S}\left(\overline{\boldsymbol{R}}^{+}\right) \cap H_{0}^{1}\left(\boldsymbol{R}^{+}\right)$, set

$$
\begin{aligned}
\|u\|_{1, t_{k}}^{2}= & \sum_{n=0}^{\infty} \lambda_{n}\left(t_{k}\right)\left|u_{n}\right|^{2} \quad\|u\|_{2, t_{k}}^{2}=\sum_{n=0}^{\infty} \lambda_{n}\left(t_{k}\right)^{2}\left|u_{n}\right|^{2} \\
\|u\|_{3, t_{k}}^{2}= & \sum_{n=0}^{\infty} \lambda_{n}\left(t_{k}\right)^{3}\left|u_{n}\right|^{2}+t_{k}{ }^{2 \kappa} \sum_{n=0}^{\infty}\left|u_{n}\right|^{2} \\
& +\left\{(K+1) t_{k}{ }^{k} / 2\right\} \sum_{n=0}^{\infty}\left(1+\lambda_{n}\left(t_{k}\right)+\lambda_{n}\left(t_{k}\right)^{2}\right)\left|u_{n}\right|^{2} .
\end{aligned}
$$

Moreover we define the space $\Phi_{3}\left(t_{k}\right)$ as the completion of $\mathcal{S}\left(\overline{\boldsymbol{R}}^{+}\right) \cap H_{0}^{1}\left(\boldsymbol{R}^{+}\right)$with respect to $\|\cdot\|_{3, t_{k}}$.

Definition 3.2. For $u(x) \in \mathcal{S}\left(\overline{\boldsymbol{R}}^{r}\right) \cap H_{0}^{1}\left(\mathbb{R}^{+}\right)$, set

$$
\begin{aligned}
{[u]_{1, t_{k}}^{2}=} & \int_{0}^{\infty}\left(2^{-1}\left|\partial_{x} u\right|^{2}+x t_{t}{ }^{\kappa}|u|^{2}\right) d x \\
{[u]_{2, t_{k}}^{2}=} & \int_{0}^{\infty}\left(4^{-1}\left|\partial_{x}{ }^{2} u\right|^{2}+x t_{k}{ }^{k}\left|\partial_{x} u\right|^{2}+x^{2} t_{k}{ }^{2 \kappa}|u|^{2}\right) d x \\
{[u]_{3, t_{k}}^{2}=} & \int_{0}^{\infty}\left(8^{-1}\left|\partial_{x}{ }^{3} u\right|^{2}+(3 / 4) x t_{k}{ }^{k}\left|\partial_{x}{ }^{2} u^{i}\right|^{2}+(3 / 2) x^{2} t_{k}{ }^{2 \kappa}\left|\partial_{x} u\right|^{2}\right. \\
& \left.+x^{3} t_{k}{ }^{3 \kappa}|u|^{2}\right) d x+\left(t_{k}{ }^{k} / 2\right)\left\{( K + 1 ) \left(\|u\|_{L^{2}}{ }^{2}+\|u\|_{1, t_{k}}{ }^{2}\right.\right. \\
& \left.\left.+\|u\|_{2, t_{k}}^{2}\right)-\left|\partial_{x} u(t, 0)\right|^{2}\right\} .
\end{aligned}
$$

Moreover we define the space $\Phi_{3}^{\prime}\left(t_{k}\right)$ as the completion of $\mathcal{S}\left(\overline{\boldsymbol{R}}^{+}\right) \cap H_{0}^{1}\left(\boldsymbol{R}^{+}\right)$with 
respect to $[\cdot]_{3, t}$.

Lemma 3.3. The two spaces $\left(\Phi\left(t_{k}\right),\|\cdot\|_{3, t_{k}}\right)$ and $\left(\Phi_{3}^{\prime}\left(t_{k}\right),[\cdot]_{3, t_{k}}\right)$ are isometric to each other. Thus from now on we also denote $\left(\Phi_{3}^{\prime}\left(t_{k}\right),[\cdot]_{3, t_{k}}\right)$ by $\left(\Phi_{3}\left(t_{k}\right)\right.$, $\left.\|\cdot\|_{3, t_{k}}\right)$. easily see

Proof. Set $u^{(N)}(x)=\sum_{n=0}^{N} u_{n} \psi_{n}\left(t_{k}, x\right)$ where $u_{n} \in C(0 \leqq n \leqq N)$. Then we can

$$
\begin{aligned}
\left\|u^{(N)}\right\|_{3, t_{k}}^{2}= & \left(H\left(t_{k}\right)^{2} u^{(N)}, H\left(t_{k}\right) u^{N}\right)_{L^{2}\left(R^{+}\right)}+t_{k}{ }^{2 \kappa}\left\|u^{(N)}\right\|_{L^{2}\left(R^{+}\right)}^{2} \\
& +2^{-1}(K+1) t_{k}{ }^{k}\left\{\left\|u^{(N)}\right\|_{L^{2}\left(R^{+}\right)}+\left(H^{\top}\left(t_{k}\right) u^{(N)}, u^{(N)}\right)_{L^{2}\left(R^{+}\right)}\right. \\
& \left.+\left\|H\left(t_{k}\right) u^{(N)}\right\|_{L^{2}\left(R^{+}\right)}^{2}\right\} .
\end{aligned}
$$

Since $\psi_{n}\left(0, t_{k}\right)=\partial_{x}{ }^{2} \psi_{n}\left(0, t_{k}\right)=0$, integrating the right hand side of the above yields $\left\|u^{(N)}\right\|_{3, t_{k}}^{2}=\left[u^{(N)}\right]_{3, t_{k}}^{2}$. Then Lemma 3.3 immediately follows from this and the fact $\left\{\psi_{n}\left(x, t_{k}\right)\right\}_{n=0}^{\infty}$ is an orthonormal system in $L^{2}\left(\mathbb{R}^{+}\right)$Q.E.D.

Next we return to the existence of $u_{\Delta}(t, x)$.

Lemma 3.4. For any partition $\Delta$ of $\left[T^{*}, T\right]$, there exists $u_{\Delta}(t, x) \subseteq L^{2}\left(\left[T^{*}, T\right]\right.$, $\Phi_{3}(T)$ ). These $\left\{u_{\Delta}(t, x)\right\}$ are bounded in $L^{2}\left(\left[T^{*}, T\right], \Phi_{3}(T)\right)$.

Proof. First we note that each $u_{\triangle}^{(k)}$ has the representation

$$
\begin{aligned}
& u_{\Delta}^{(k)}(t, x)=\sum_{n=0}^{\infty} \exp \left\{-\lambda_{n}\left(t_{k}\right)\left(1-t_{k}\right)\right\} d_{n}^{(k)} \phi_{n}\left(t_{k}, x\right) \\
& d_{n}^{(k)}=\int_{0}^{\infty} u_{\Delta}^{(k-1)}\left(t_{k}, x\right) \phi_{n}\left(t_{k}, x\right) d x .
\end{aligned}
$$

Combining this with Lemma $3.3, u_{\Delta}^{(i)}(t, \cdot) \in \Phi_{3}\left(t_{k}\right)$ and

$$
\left\|u_{\Delta}^{(k)}\left(t_{k}, \cdot\right)\right\|_{3, t_{k}}=\left\|u_{\Delta}^{(k)}(t, \cdot)\right\|_{3, t_{k}} \quad \text { for any } t \in\left[t_{k+1}, t_{k}\right] .
$$

Now according to Definition 3.2 and Lemma 3.3

$$
\left\|u_{\Delta}^{(k)}\left(t_{k+1}, \cdot\right)\right\|_{3, t_{k+1}} \leqq\left\|u_{\Delta}^{(k)}\left(t_{k+1}, \cdot\right)\right\|_{3, t_{k}} .
$$

Moreover, by the definition of $u_{\Delta}^{(k)}$

$$
\left\|u_{\Delta}^{(k)}\left(t_{k+1}, \cdot\right)\right\|_{3, t_{k+1}}=\left\|u_{\Delta}^{(k+1)}\left(t_{k+1}, \cdot\right)\right\|_{3, t_{k+1}} .
$$

Hence combining (3.11) (3.13) and taking account of $\|\cdot\|_{q, T} \sim\|\cdot\|_{q, T}$, there is a constant $c>0$ such that

$$
\left\|u_{\Delta}(t, \cdot)\right\|_{3, T} \leqq c\|h(T, \cdot)\|_{2, T} \quad\left(T^{*} \leqq t \leqq T\right) \quad \text { for any } \Delta .
$$

Q.E.D.

Lemma 3.5. There is a constant $c>0$ such that 


$$
\left\|u_{\Delta}(t, \cdot)\right\|_{3, T} \geqq c\left\|u_{\Delta}(t, \cdot)\right\|_{w_{2}^{3}\left(R^{+}\right)} \quad\left(T^{*} \leqq t \leqq T\right) \quad \text { for any } \Delta .
$$

Proof. This is almost clear from Definition 3.2.

Theorem 3.6. There is a solution $u(t, x) \in L^{2}\left(\left[T^{*}, T\right], \Phi_{3}(T)\right) \cap \mathcal{E}_{L^{2}}^{1,3}\left(\left(T^{*}, T\right)\right.$ $\left.\times \boldsymbol{R}^{+}\right)$to (3.6).

Here

$$
\begin{gathered}
\mathcal{E}_{L^{2}}^{p, q}\left(\left(T^{*}, T\right) \times \boldsymbol{R}^{+}\right)=\left\{u \in \mathscr{D}^{\prime}\left(\left(T^{*}, T\right) \times \mathbb{R}^{+}\right) ; \partial_{t}^{j} \partial_{x}^{k} u(t, x) \in L^{2}\left(\left(T^{*}, T\right) \times \boldsymbol{R}^{+}\right)\right. \\
(j \leqq p, k \leqq q-2 j)\}
\end{gathered}
$$

for nonnegative integers $p, q(2 p<q)$ and we will see later in Corollary 4.4 that this $u(t, x)$ is unique.

Proof of Theorem 3.6. By Lemma 3.4, $\left\{u_{\Delta}(t, x)\right\}$ is uniformly bounded in the Hilbert space $L^{2}\left(\left[T^{*}, T\right], \Phi_{s}(T)\right)$. Hence there exists

$$
u(t, x) \in L^{2}\left(\left[T^{*}, T\right], \Phi_{3}(T)\right)
$$

and a sequence of functions $\left\{u_{\jmath}(t, x)\right\} \subset\left\{u_{\Delta}(t, x)\right\}$ such that $u_{\jmath}(t, x) \rightarrow u(t, x)$ $(j \rightarrow \infty)$ weakly in $L^{2}\left(\left[T^{*}, T\right], \Phi_{3}(T)\right)$. On the other hand, using the definition of $u_{\Delta}(t, x)$, each $u_{\jmath}(t, x)$ is the approximate solution to the equation of (3.6). Hence $u(t, x)$ satisfies the same equation in the distribution sense.

Now by Lemma 3.5, the argument which led to (3.16) yields

$$
u(t, x) \in \mathcal{E}_{L^{2}}^{0,3}\left(\left(T^{*}, T\right) \times \mathbb{R}^{+}\right) .
$$

Combining (3.16), (3.17) with the equation of (3.6) and reminding the definition of $\Phi_{3}(T)$, we have

$$
u(t, x) \in L^{2}\left(\left[T^{*}, T\right], \Phi_{3}(T)\right) \cap \mathcal{E}_{L^{2}}^{1,3}\left(\left(T^{*}, T\right) \times \mathbb{R}^{+}\right) .
$$

Thus $u(t, x)$ satisfies the equation in the $L^{2}$ strong sense and it has the traces to $t=0$ and $x=0$.

Finally, to see that $u(t, x)$ satisifes the boundary condition and the initial condition of (3.6) is a routine argument (see Mizohata [3] p. 333). Q.E.D.

\section{§4. Prooi of IVain Theorem (part 2)}

In this section, we study the regularity and decay at infinity of the principal term $V_{0}=\psi(t, x)$ of the expansion (2.11).

To begin with, we generalize (3.6) as follows:

$$
\left\{\begin{array}{l}
i \partial_{t} u=(-1 / 2) \partial_{2}^{2} u+x t^{r} u+f(t, x) \quad \text { in }\left(T^{*}, T\right) \times \mathbb{R}^{+} \\
\left.u\right|_{x=0}=0 \\
\left.u\right|_{t=T}=u_{n}^{\prime}(x) .
\end{array}\right.
$$


Lemma 4.1. Let $f(t, x) \in L^{2}\left(\left[T^{*}, T\right], \Phi_{3}(T)\right)$ and $u_{0}(x) \in \mathcal{S}\left(\overline{\boldsymbol{R}}^{+}\right) \cap H_{0}^{1}\left(\boldsymbol{R}^{+}\right)$then there exists a solution

$$
u(t, x) \in L^{2}\left(\left[T^{*}, T\right], \Phi_{3}(T)\right) \cap \mathcal{E}_{L^{2}}^{1,3}\left(\left(T^{*}, T\right) \times \boldsymbol{R}^{+}\right)
$$

to (4.1) which satisfies

$$
\begin{aligned}
& {\left[\int_{T *}^{T}\left(\|u(t, \cdot)\|_{\Phi_{3}(T)}\right)^{2} d t\right]^{1 / 2}=\|u(t, x)\|_{L^{2}\left([T *, T], \Phi_{3}(T)\right)}} \\
& \quad \leqq C\left\{\left(T-T^{*}\right)^{1 / 2}\left\|u_{0}\right\|_{\Phi_{3}(T)}+\left(T-T^{*}\right)\|f(t, x)\|_{L^{2}\left([T *, T], \Phi_{3}(T)\right)}\right\}
\end{aligned}
$$

where $C$ is a constant depending only on $T^{*}, T$.

Proof. As in part 1, we apply Cauchy's method. Take a partition $\Delta: T^{*}$ $=t_{m}<t_{m-1}<\cdots<t_{1}<t_{0}=T$ and define $u_{\Delta}(t, x)=u_{\Delta}^{(k)}(t, x), \quad\left(k=0,1, \cdots, m-1 ; t_{k+1}\right.$ $\left.\leqq t \leqq t_{k}\right)$ by

$$
\left[\begin{array}{l}
i \partial_{t} u_{\Delta}^{(k)}=-(1 / 2) \partial_{x}^{2} u_{\Delta}^{(k)}+x t_{k}{ }^{k} u_{\Delta}^{(k)}+f(t, x), \quad\left(t_{k+1}<t<t_{k}, x>0\right) \\
\left.u_{\Delta}^{(k)}\right|_{x=0}=0 \quad\left(t_{k+1} \leqq t \leqq t_{k}\right) \\
u_{\Delta}^{(k)}\left(t_{k}, x\right)=u_{\Delta}^{(k-1)}\left(t_{k}, x\right) \quad(x \geqq 0)
\end{array}\right.
$$

such that $u_{\Delta}^{(-1)}\left(t_{0}, x\right)=u_{0}(x)$.

Then, using the eigenfunctions $\left\{\psi_{n}\left(t_{k}, x\right)\right\}_{n=0}^{\infty}$, and eigenvalues $\left\{\lambda_{n}\left(t_{k}\right)\right\}_{n=0}^{\infty}$ of the operator $H\left(t_{k}\right)=-(1 / 2) \partial_{x}^{2}+x t_{k}{ }^{k}$, we have

$$
\begin{aligned}
u_{\Delta}^{(k)}(t, x)= & \sum_{n=0}^{\infty} \exp \left\{-i \lambda_{n}\left(t_{k}\right)\left(t-t_{k}\right)\right\} d_{n}^{(k)} \psi_{n}\left(t_{k}, x\right) \\
& +\sum_{n=0}^{\infty} \int_{t}^{t_{k}} \exp \left\{-i \lambda_{n}\left(t_{k}\right)(t-s)\right\} i f_{n}^{(k)}(s) d s \phi_{n}\left(t_{k}, x\right)
\end{aligned}
$$

where

$$
d_{n}^{(k)}=\int_{0}^{\infty} u_{\Delta}^{(k-1)}\left(t_{k}, x\right) \phi_{n}\left(t_{k}, x\right) d x, \quad u_{\Delta}^{(-1)}\left(t_{0}, x\right)=u_{0}(x)
$$

and $f_{n}^{(k)}(t)=\int_{0}^{\infty} f(t, x) \psi_{n}\left(t_{k}, x\right) d x$. By applying Minkowski's inequality, we can easily see

$$
\left\|u_{L}^{(k)}(t, x)\right\|_{3, t_{k}} \leqq\left\|u_{L}^{(k)}\left(t_{k}, x\right)\right\|_{3, t_{k}}+\int_{t}^{t_{k}}\|f(s, \cdot)\|_{3, t_{k}} d s .
$$

Now following the same argument as in the proof of Lemma 3.4, We have

Thus

$$
\left\|u_{\Delta}(t, x)\right\|_{3, t_{k}} \leqq\left\|u_{0}(x)\right\|_{\Phi_{3}(T)}+\int_{t}^{T}\|f(s, \cdot)\|_{\Phi_{3}(T)} d s .
$$

$$
\begin{aligned}
& {\left[\int_{T^{*}}^{T}\left\|u_{L}(t, x)\right\|_{\Phi_{3}(T)}^{2} d t\right]^{1 / 2} } \leqq C\left\{\left(T-T^{*}\right)^{1 / 2}\left\|u_{0}(x)\right\|_{\Phi_{3}(T)}\right. \\
&\left.+\left(T-T^{*}\right)\left[\int_{T_{*}^{*}}^{T}\|f(s, \cdot)\|_{\Phi_{3}(T)}^{\mathcal{D}_{(}} d s\right]^{1 / 2}\right\} .
\end{aligned}
$$


The rest of the proof is the same as that of Theorem 3.6.

Q.E.D.

Remark. Later in Corollary 4.4, we show that this solution is unique.

To derive the energy inequality for (4.1), we need the following lemma.

Lemma 4.2. Let $u(t, x) \in L^{2}\left(\left[T^{*}, T\right], \Phi_{3}(T)\right)$ be the solution to (4.1), where $f(t, x) \in C^{0}\left(\left[T^{*}, T\right], L^{2}\left(\boldsymbol{R}^{+}\right)\right) \cap L^{2}\left(\left[T^{*}, T\right], \Phi_{3}(T)\right) \quad$ and $\quad u_{0}(x) \in \mathcal{S}\left(\overline{\boldsymbol{R}}^{+}\right)$. Then $\|u(t, \cdot)\|,\|x u(t, \cdot)\|,\left\|\partial_{x}^{2} u(t, \cdot)\right\|,\left\|u^{\prime}(t, \cdot)\right\| \in C^{0}\left(\left[T^{*}, T\right]\right)$.

Notation. Hereafter, for simplicity, we denote the $L^{2}\left(\boldsymbol{R}^{+}\right)$norm by $\|\cdot\|$, and the differential with respect to $t$ by " $"$ ".

Proof of Lemma 4.2. From the assumptions, $\partial_{t} u(t, x) \in L^{2}\left(\left[T^{*}, T\right] \times \boldsymbol{R}^{+}\right)$. Then by Radon Nikodym's theorem

$$
u(t, x)-u\left(t^{\prime}, x\right)=\int_{t^{\prime}}^{t} \partial_{s} u(s, x) d s \quad \text { for a. e. } x .
$$

So we have $u(t, x) \in C^{0}\left(\left[T^{*}, T\right], L^{2}\left(\boldsymbol{R}^{+}\right)\right)$. Therefore

$$
\|u(t, \cdot)\| \in C^{0}\left(\left[T^{*}, T\right]\right) .
$$

To prove $\|x u(t, \cdot)\| \in C^{0}\left(\left[T^{*}, T\right]\right)$, put $(u)_{\delta}(t, x)=\left(\phi_{\delta}^{(t)} * u\right)(t, x)$ where $\phi_{\delta}$ is a mollifier such that

$$
\begin{array}{r}
\phi_{\delta}(t)=\frac{1}{\delta} \phi(t / \delta), \quad \phi(t) \geqq 0, \quad \phi(t) \in C_{0}^{\infty}(\boldsymbol{R}), \\
\operatorname{supp} \phi \subset\{t|| t \mid \leqq 1\}, \quad \int_{\boldsymbol{R}} \phi(t) d t=1 .
\end{array}
$$

Now let $u_{\Delta}(t, x)$ be the one defined in the proof of Lemma 4.1. Then by integration by parts,

$$
\begin{aligned}
& \left|\frac{d}{d t}\left\|x\left(u_{\Delta}\right)_{\delta}(t, \cdot)\right\|^{2}\right| \\
& \quad \leqq 2\left\|\partial_{x}\left(u_{\Delta}\right)_{\delta}(t, \cdot)\right\| \cdot\left\|x\left(u_{\Delta}\right)_{\delta}(t, \cdot)\right\|+2\left\|x f_{\delta}(t, \cdot)\right\| \cdot{ }_{\|}^{\prime} x\left(u_{\Delta}\right)_{\delta}(t, \cdot) \| \cdot
\end{aligned}
$$

Thus

$$
\begin{aligned}
& \left\|\frac{d}{d t}\right\| x\left(u_{\Delta}\right)_{\delta}(t, \cdot)\|\|_{L^{2}([T *, T])} \\
& \leqq\left\|\partial_{x}\left(u_{\Delta}\right)_{\delta}(t, x)\right\|_{L^{2}\left([T *, T] \times R^{+}\right)}+\left\|x f_{\delta}(t, x)\right\|_{L^{2}\left([T *, T] \times \boldsymbol{R}^{+}\right)} \\
& \leqq\left\|\partial_{x} u_{\Delta}(t, x)\right\|_{L^{2}\left([T *, T] \times R^{+}\right)}+\|x f(t, x)\|_{L^{2}\left([T *, T] \times R^{+}\right)} \leqq M,
\end{aligned}
$$

where $M$ is a constant independent of $\Delta$. On the other hand,

$$
\int_{T *}^{T}\left(\left\|x\left(u_{\Delta}\right)_{\delta}(t, \cdot)\right\|-\left\|x u_{\Delta}(t, \cdot)\right\|\right)^{2} d t \longrightarrow 0 \quad(\delta \rightarrow 0) .
$$


Therefore taking an appropriate subsequence $\left\{\delta^{\prime}\right\} \subset\{\delta\}$,

$$
\frac{d}{d t}\left\|x\left(u_{\Delta}\right)_{\delta^{\prime}}(t, \cdot)\right\| \longrightarrow \frac{d}{d t}\left\|x u_{\Delta}(t, \cdot)\right\| \quad\left(\delta^{\prime} \rightarrow 0\right) \quad \text { in } \mathscr{D}^{\prime}\left(\left(T^{*}, T\right)\right)
$$

and

$$
\left\|\frac{d}{d t}\right\| x u_{\Delta}(t, \cdot)\|\|_{L^{2}\left(\left[T^{*}, T\right]\right)} \leqq M \quad \text { for any } \Delta .
$$

Here $d / d t$ is the distribution derivative. Next, we will show

$$
\left\|x u_{\Delta}(t, \cdot)\right\| \longrightarrow\|x u(t, \cdot)\| \quad(|\Delta| \rightarrow 0) \quad \text { in } \mathscr{D}^{\prime}\left(\left(T^{*}, T\right)\right) .
$$

From (4.2), $\left\|x^{1 / 2} u_{\Delta}(t, \cdot)\right\|$ is uniformly bounded on $\left[T^{*}, T\right]$ with respect to $\Delta$. By $(4.2)^{\prime}, \partial_{t} x^{1 / 2} u_{\Delta}(t, x)$ is uniformly bounded in $L^{2}\left(\left[T^{*}, T\right] \times \boldsymbol{R}^{+}\right)$with respect to $\Delta$, and by Radon Nikodym's theorem we have

$$
\left\|x^{1 / 2} u_{\Delta}\left(t^{\prime}, \cdot\right)-x^{1 / 2} u_{\Delta}(t, \cdot)\right\|^{2} \leqq\left|t^{\prime}-t\right| \int_{T *}^{T}\left\|\partial_{s} x^{1 / 2} u_{\Delta}(s, \cdot)\right\|^{2} d s .
$$

Thus $x^{1 / 2} u_{\Delta}(t, x)$ is uniformly bounded and equi-continuous in $C^{0}\left(\left[T^{*}, T\right], L^{2}\left(\boldsymbol{R}^{+}\right)\right)$ with respect to $\Delta$. So for an appropriate sequence $\left\{\Delta^{\prime}\right\} \subset\{\Delta\}$,

$$
\operatorname{Sup}_{T * \leq t \leq T}\left\|x^{1 / 2} u_{\Delta^{\prime}}(t, \cdot)-x^{1 / 2} u(t, \cdot)\right\| \longrightarrow 0 \quad\left(\mid \Delta^{\prime} ; \rightarrow 0\right)
$$

and

$$
\begin{aligned}
& \left|\int_{T^{*}}^{T}\left(\left\|x u_{\Delta^{\prime}}(t, \cdot)\right\|-\|x u(t, \cdot)\|\right) \cdot \phi(t) d t\right| \\
& \leqq\left(\int_{T^{*}}^{T}|\phi(t)|^{2} d t\right)^{1 / 2}\left\{\int_{T^{*}}^{T} \int_{R^{+}} x^{1 / 2}\left(u_{\Delta^{\prime}}(t, x)-u(t, x)\right) x^{3 / 2}\right. \\
& \cdot\left(\overline{u_{\Delta^{\prime}}(t, x)}-\overline{u(t, x))} d x d t\right\}^{1 / 2} \\
& \leqq\left(\int_{T^{*}}^{T}|\phi(t)|^{2} d t\right)^{1 / 2}\left\{\int_{T^{*}}^{T} \int_{R^{+}}\left|x^{1 / 2}\left(u_{\Delta^{\prime}}(t, x)-u(t, x)\right)\right|^{2} d x d t\right. \\
& \left.\quad \times \int_{T^{*}}^{T} \int_{R^{+}}\left|x^{3 / 2}\left(u_{\Delta^{\prime}}(t, x)-u(t, x)\right)\right|^{2} d x d t\right\}^{1 / 4} \\
& \leqq\left(\int_{T *}^{T}|\phi(t)|^{2} d t\right)^{1 / 2}\left(T-T^{*}\right)^{1 / 4}\left\{\operatorname{Sup} \| x^{1 / 2} u_{\Delta^{\prime}}^{\left.(t, \cdot)-x^{1 / 2} u(t, \cdot) \|^{2}\right\}^{1 / 4}}\right. \\
& \quad \times\left\{2 \int_{T^{*}}^{T} \int_{R^{+}}\left|x^{3 / 2} u_{\Delta^{\prime}}\right|^{2}+\left|x^{3 / 2} u\right|^{2} d x d t\right\}^{1 / 4} \stackrel{\left(\left|\Delta^{\prime}\right| \rightarrow 0\right)}{\longrightarrow} 0,
\end{aligned}
$$

for any $\phi \in C_{0}^{\infty}\left(\left(T^{*}, T\right)\right)$. Hence we have proved (4.4). Since (4.3) and (4.4) imply

$$
\frac{d}{d t}\|x u(t, \cdot)\| \in L^{2}\left(\left[T^{*}, T\right]\right),
$$

we have $\|x u(t, \cdot)\| \in C^{0}\left(\left[T^{*}, T\right]\right)$. The same argument can also be applied to 
prove $\left\|\partial_{x}^{2} u(t, \cdot)\right\| \in C^{0}\left(\left[T^{*}, T\right]\right)$. Finally, by using the equation of $(4.1),\left\|u^{\prime}(t, \cdot)\right\|$ $\in C^{0}\left(\left[T^{*}, T\right]\right)$.

Q.E.D.

Relying on Lemma 4.2, we will give the following energy estimate for the solution to (4.1).

Lemma 4.3 (Energy Inequality). Let $f(t, x) \in L^{2}\left(\left[T^{*}, T\right], \Phi_{3}(T)\right), \partial_{t} f(t, x)$ $\in L^{2}\left(\left[T^{*}, T\right], L^{2}\left(\boldsymbol{R}^{+}\right)\right), u_{0}(x)=u(T, x) \in \mathcal{S}\left(\overline{\boldsymbol{R}}^{+}\right)$and let $u(t, x) \in L^{2}\left(\left[T^{*}, T\right], \Phi_{3}(T)\right)$ be the solution to (4.1). Then we have the following energy inequality:

$$
\begin{aligned}
& \|u(t, \cdot)\|+t^{\kappa}\|x u(t, \cdot)\|+\left\|u^{\prime}(t, \cdot)\right\| \\
& \leq\|u(T, \cdot)\|+2\left(T^{2} / t\right)^{x}\|x u(T, \cdot)\|+(T / t)^{\kappa}\left\|\partial_{x}^{2} u(T, \cdot)\right\| \\
& \quad+2(T / t)^{x}\|f(T, \cdot)\|+\|f(t, \cdot)\|+\int_{t}^{T}\{\|f(s, \cdot)\| \\
& \left.\quad+2 \kappa(1 / s)(s / t)^{\kappa}\|f(s, \cdot)\|+2(s / t)^{x}\left\|f^{\prime}(s, \cdot)\right\|\right\} d s \quad \text { for a.e. } t \in\left[T^{*}, T\right] .
\end{aligned}
$$

Proof. Since

$$
\frac{d}{d t}\|u(t, \cdot)\|^{2}=2 \operatorname{Im} \int_{0}^{\infty} f(t, x) \overline{u(t, x)} d x,
$$

we have $|(d / d t)\|u(t, \cdot)\|| \leqq\|f(t, \cdot)\|$. Thus

$$
\|u(t, \cdot)\| \leqq\|u(T, \cdot)\|+\int_{t}^{T}\|f(s, \cdot)\| d s .
$$

By integrating by parts,

$$
\begin{aligned}
& \int_{0}^{\infty} i \partial_{t} u \cdot x \bar{u} d x=\int_{0}^{\infty}\left\{(-1 / 2) \partial_{x}^{2} u+x t^{\kappa} u+f\right\} x \bar{u} d x \\
& \quad=\int_{0}^{\infty}(1 / 2) \partial_{x} u \cdot x \overline{\partial_{x} u} d x+\int_{0}^{\infty}(1 / 2) \partial_{x} u \cdot \bar{u} d x+\int_{0}^{\infty}|x u|^{2} t^{n} d x+\int_{0}^{\infty} f x \bar{u} d x .
\end{aligned}
$$

Hence,

$$
\begin{aligned}
(1 / 2) & \int_{0}^{\infty} x\left|\partial_{x} u\right|^{2} d x+\int_{0}^{\infty}|x u|^{2} t^{x} d x \\
& =\int_{0}^{\infty} i \partial_{t} u \cdot x \bar{u} d x-\int_{0}^{\infty}(1 / 2) \partial_{x} u \cdot \bar{u} d x-\int_{0}^{\infty} f x \bar{u} d x .
\end{aligned}
$$

Taking the real parts of the right hand side, we have

$$
t^{\kappa} \int_{0}^{\infty}|x u|^{2} d x \leqq\left\|\partial_{t} u(t, \cdot)\right\|\|x u(t, \cdot)\|+\|f(t, \cdot)\|\|x u(t, \cdot)\| .
$$

Thus

$$
t^{\kappa}\|x u(t, \cdot)\| \leqq\left\|\partial_{t} u(t, \cdot)\right\|+\|f(t, \cdot)\| .
$$

Now let $\phi_{\delta}(t)$ be the mollifier used in the proof of Lemma 5.3 and put $u_{\delta}(t, x)$ $=\left(\phi_{\delta} * u\right)(t, x)$. Then 


$$
i \partial_{t} u_{\delta}=-(1 / 2) \partial_{x}^{2} u_{\delta}+x t^{\kappa} u_{\delta}+\tilde{f}(t, x)
$$

where $\tilde{f}(t, x)=x\left[\phi_{\delta^{*}}, t^{\kappa}\right] u+f_{\delta}(t, x),\left[\phi_{\delta^{*}}, t^{\kappa}\right] u=\phi_{\delta^{*}} * t^{\kappa} u-t^{\kappa} u_{\hat{\delta}}$. Since

we have

$$
i \partial_{t} u_{\delta}^{\prime}=-(1 / 2) \partial_{x}^{2} u_{\delta}{ }^{\prime}+x t^{\kappa} u_{\delta}{ }^{\prime}+\kappa x t^{\kappa-1} u_{\delta}+\tilde{f}^{\prime}(t, x),
$$

$$
\begin{aligned}
& \frac{d}{d t}\left\|u_{\delta}^{\prime}\right\|^{2}=2 \operatorname{Re} \int_{0}^{\infty} \partial_{t} u_{\delta}{ }^{\prime} \cdot \bar{u}_{\delta}{ }^{\prime} d x \\
& \quad=2 \operatorname{Im} \int_{0}^{\infty}\left\{-(1 / 2) \partial_{x}^{2} u_{\delta}^{\prime}+x t^{\kappa} u_{\delta}+\kappa x t^{\kappa-1} u_{\delta}+\tilde{f}^{\prime}\right\} \bar{u}_{\delta}^{\prime} d x \\
& \quad=2 \operatorname{Im} \int_{0}^{\infty}\left(\kappa x t^{\kappa-1} u_{\delta}+\tilde{f}^{\prime}\right) \bar{u}_{\delta}^{\prime} d x .
\end{aligned}
$$

So

$$
\left|\frac{d}{d t}\left\|u_{\delta}{ }^{\prime}(t, \cdot)\right\|\right| \leqq\left\|\kappa x t^{\kappa-1} u_{\delta}(t, \cdot)\right\|+\left\|\tilde{f}^{\prime}(t, \cdot)\right\|
$$

From (4.6)

Thus we have

$$
t^{\kappa}\left\|x u_{\tilde{o}}(t, \cdot)\right\| \leqq\left\|\partial_{t} u_{\delta}(t, \cdot)\right\|+\|\tilde{f}(t, \cdot)\|
$$

$$
\left|\frac{d}{d t}\left\|u_{\delta}^{\prime}(t, \cdot)\right\|\right| \leqq(\kappa / t)\left\|u_{\delta}^{\prime}(t, \cdot)\right\|+(\kappa / t)\|\tilde{f}(t, \cdot)\|+\left\|\tilde{f}^{\prime}(t, \cdot)\right\| \cdot
$$

Applying the comparison theorem, we have

$$
\begin{aligned}
\left\|u_{\delta}{ }^{\prime}(t, \cdot)\right\| \leqq & (T / t)^{\kappa}\left\|u_{\delta}{ }^{\prime}(T, \cdot)\right\|+\int_{t}^{T}(1 / s)(s / t)^{\kappa}\left(\kappa\|\tilde{f}(s, \cdot)\|+s\left\|\tilde{f}^{\prime}(s, \cdot)\right\|\right) d s \\
\leqq & (T / t)^{\kappa}\left\|u_{\delta}{ }^{\prime}(T, \cdot)\right\|+\int_{t}^{T}(1 / s)(s / t)^{\kappa} \cdot\left\{\kappa\left\|x\left[\phi_{\delta^{*}}, s^{\kappa}\right] u\right\|\right. \\
& \left.+\kappa\left\|f_{\delta}(s, \cdot)\right\|+s\left(\left\|x \partial_{s}\left[\phi_{\delta^{*}}, s^{\kappa}\right] u\right\|+\left\|\partial_{s} f_{\delta}(s, \cdot)\right\|\right)\right\} d s .
\end{aligned}
$$

Since

$$
x\left[\phi_{\delta^{*}}, s^{\aleph}\right] u(s, \cdot)=\phi_{\delta^{*}}\left(s^{\kappa} x u\right)-s^{\kappa} \phi_{\delta^{*}} x u=\int_{R} \phi_{\delta}(s-\sigma)\left(\sigma^{\kappa}-s^{k}\right) x u(\sigma, x) d \sigma,
$$

we have

$$
\begin{aligned}
\left\|x\left[\phi_{\delta^{*}}, s^{\kappa}\right] u(s, \cdot)\right\| & \leqq\left\{\int_{R} \phi_{\delta}(s-\sigma)\left|\sigma^{\kappa}-s^{\kappa}\right|^{2}\|x u(\sigma, \cdot)\|^{2} d \sigma\right\}^{1 / 2} \\
& \leqq C\left(T, T^{*}\right) \cdot\left(\delta^{2} \int_{R} \phi(t) t^{2}\|x u(s-\delta t, \cdot)\|^{2} d t\right)^{1 / 2}
\end{aligned}
$$

$\longrightarrow 0 \quad(\delta \rightarrow 0)$ uniformly with respect to $s \in[t, T]$.

Similarly, we have

$$
\int_{t}^{T}\left\|x \partial_{s}\left[\phi_{\delta^{*}}, s^{\kappa}\right] u(s, \cdot)\right\| d s \longrightarrow 0 \quad(\delta \rightarrow 0) .
$$

Moreover, taking account of the assumption and Lemma 4.2, it is clear 


$$
\begin{aligned}
& \left\|u_{\dot{\delta}}{ }^{\prime}(t, \cdot)\right\| \longrightarrow\left\|u^{\prime}(t, \cdot)\right\| \quad(\delta \rightarrow 0) \quad \text { for a. e. } t \in\left[T^{*}, T\right] . \\
& \left\|u_{\delta^{\prime}}(T, \cdot)\right\|^{2} \leqq \int_{R} \phi_{\dot{\delta}}(T-\tau)\left\|u^{\prime}(\tau, \cdot)\right\|^{2} d \tau \longrightarrow\left\|u^{\prime}(T, \cdot)\right\|^{2} \quad(\delta \rightarrow 0),
\end{aligned}
$$

and $\int_{t}^{T}\left\|f_{\delta}(s, \cdot)-f(s, \cdot)\right\| d s \rightarrow 0(\delta \rightarrow 0)$. Hence by letting $\delta \rightarrow 0,(4.7)_{\delta}$ implies

$$
\begin{aligned}
\left\|u^{\prime}(t, \cdot)\right\| \leqq & (T / t)^{\kappa}\left\|u^{\prime}(T, \cdot)\right\|+\int_{t}^{T}(1 / s)(s / t)^{\kappa}\left(\kappa\|f(s, \cdot)\|+s\left\|\partial_{s} f(s, \cdot)\right\|\right) d s \\
\leqq & (T / t)^{\kappa}\left((1 / 2)\left\|\partial_{x}^{2} u(T, \cdot)\right\|+\left\|x T^{\kappa} u(T, \cdot)\right\|+\|f(T, \cdot)\|\right) \\
& +\int_{t}^{T}(1 / s)(s / t)^{\kappa}\left(\kappa\|f(s, \cdot)\|+s\left\|f^{\prime}(s, \cdot)\right\|\right) d s .
\end{aligned}
$$

Combining (4.5), (4.6) and (4.7), we have the desired inequality.

Q.E. D.

Next Corollary 4.4 follows from (4.5).

Corellary 4.4 (Uniqueness). Let $u(t, x) \in L^{2}\left(\left[T^{*}, T\right] \times \boldsymbol{R}^{+}\right)$be the solution to (4.1) such that $\partial_{x}^{2} u(t, x), x u(t, x) \in L^{2}\left(\left[T^{*}, T\right] \times \boldsymbol{R}^{+}\right)$. Then $u(t, x)$ is a unique solution to (4.1).

Next using Lemma 4.3, we will prove the higher order regularity of the solution $u(t, x)$ to (3.5) with respect to $t$. But, for a while, we restrict the the domain of $t$ to the bounded interval $\left[T^{*}, T\right]$.

Now, taking a positive integer $m$ large enough, we seek the solution $\tilde{u}(t, x)$ to (3.6) in the form

$$
\begin{aligned}
\tilde{u}(t, x)= & u_{0}(x)+(t-T) u_{1}(x)+\cdots+\frac{(t-T)^{m-1}}{(m-1) !} u_{m-1}(x) \\
& +\int_{t}^{T} \frac{(t-s)^{m-1}}{(m-1) !} v(s, x) d s \quad\left((t, x) \in\left[T^{*}, T\right] \times \overline{\boldsymbol{R}}^{+}\right) .
\end{aligned}
$$

Then we have the following.

Lemma 4.5. In order that (4.8) is the solution to (3.6), it is necessary and sufficient that the following conditions (4.9) and (4.10) hold. Namely,

$$
\left\{\begin{array}{l}
u_{0}(x)=h(T, x) \\
u_{1}(x)=(1 / i)\left\{(-1 / 2) \partial_{x}^{2} u_{0}(x)+x T^{\kappa} u_{0}(x)\right\} \\
u_{p}(x)=(1 / i)\left\{(-1 / 2) \partial_{x}^{2} u_{p-1}(x)+\sum_{l=0}^{r}\left(\begin{array}{c}
p-1 \\
l
\end{array}\right) x \partial_{T}^{l} T^{\mathfrak{r}} u_{p-l-1}(x)\right\} \\
\quad 2 \leqq p \leqq m-1, \quad \gamma=\min (p-1, \kappa) \\
u_{p}(0)=0 \quad 0 \leqq p \leqq m-1
\end{array}\right.
$$




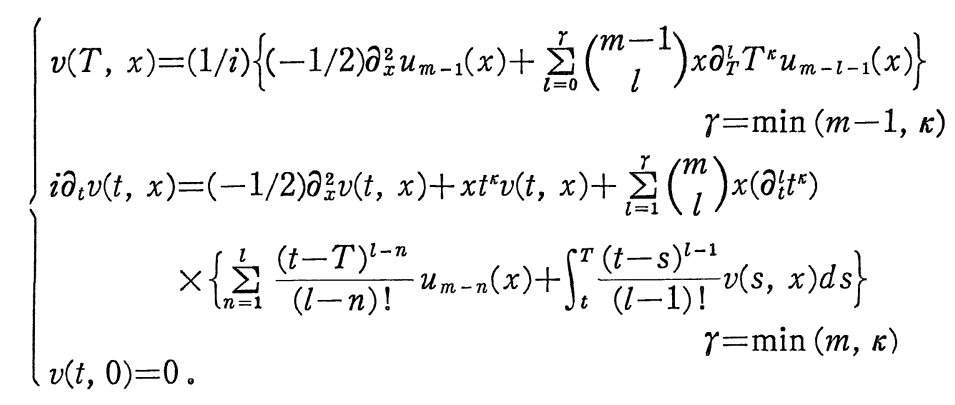

Proof. From (3.6), it follows that

$$
\left[\begin{array}{l}
\left.\partial_{t}^{p}\left(i \partial_{t} \tilde{u}+(1 / 2) \partial_{x}^{2} \tilde{u}-x t^{\kappa} \tilde{u}\right)\right|_{t=T}=0 \quad 0 \leqq p \leqq m-1 \\
\partial_{t}^{m}\left(i \partial_{t} \tilde{u}+(1 / 2) \partial_{x}^{2} \tilde{u}-x t^{\kappa} \tilde{u}\right)=0
\end{array}\right.
$$

Substituting (4.8) into the above equations, we obtain (4.9), (4.10)。 Conversely, from the above equations we can easily see that $\tilde{u}(t, x)$ is the solution to (3.6) if (4.9), (4.10) are valid.

Remark. Since the equation in (3.2) is non characteristic with respect to $x=0$, we can assume that $h(t, x)$ is flat at $x=0$ by modifying $w(t, x)$. Then the conditions (4.9) and (4.10) are valid.

Now we prove the existence of the solution to (4.10) by the successive approximation.

Define $\left\{v_{j}(t, x)\right\}_{0}^{\infty}$ inductively by

$$
\left\{\begin{array}{c}
i \partial_{t} v_{\jmath}+(1 / 2) \partial_{x}^{2} v_{\jmath}-x t^{\kappa} v_{\jmath}=\sum_{l=1}^{r}\left(\begin{array}{c}
m \\
l
\end{array}\right) x\left(\partial_{t}^{l} t^{\kappa}\right) \\
\quad \times\left\{\sum_{n=1}^{l} \frac{(t-T)^{l-n} !}{(l-n) !} u_{m-n}(x)+\int_{t}^{T} \frac{(t-s)^{l-1}}{(l-1) !} v_{j-1}(s, x) d s\right\} \\
v_{\jmath}(T, x)=u_{m}(x) \\
v_{j}(t, 0)=0 \quad j=1,2, \cdots, \quad v_{0}(t, x) \equiv 0
\end{array}\right.
$$

As for the solvability of each (4.11.j), we need the following Lemma 4.6.

Lemma 4.6. Let $\Phi_{2}(T)$ be the completion of $S\left(\overline{\mathbb{R}}^{+}\right) \cap H_{0}^{1}\left(\boldsymbol{R}^{+}\right)$with respect to the norm $\|\cdot\|_{2 . T}$. We can find the solution $v_{j}(t, x) \in L^{2}\left(\left[T^{*}, T\right], \Phi_{2}(T)\right)$ to (4.11.j), $(j=1,2, \cdots)$ 。

Proof. We will prove this by induction on $j$. By Lemma 4.1, this is true for the case $j=1$. Assume $v_{j-1} \in L^{2}\left(\left[T^{*}, T\right], \Phi_{2}(T)\right.$ ). Denote by $g(t, x)$ the right hand side of the first equation of (4.11.j). Then $g(t, x) \in C^{0}\left(\left[T^{*}, T\right], L^{2}\left(\mathbb{R}^{+}\right)\right)$ 
and $g^{\prime}(t, x)=\partial_{t} g(t, x) \in L^{2}\left(\left[T^{*}, T\right], L^{2}\left(\boldsymbol{R}^{+}\right)\right)$exists for almost everywhere $t \in$ $\left[T^{*}, T\right]$.

Let $\psi_{n}(T, x)=\psi_{n}(x) n=0,1, \cdots$ be the orthonormal system defined by (3.8) and expand $g(t, x)$ as follows:

$$
g(t, x)=\sum_{n=0}^{\infty} g_{n}(t) \psi_{n}(x), \quad \text { where } g_{n}(t)=\int_{0}^{\infty} g(t, x) \psi_{n}(x) d x .
$$

Let $g_{N}(t, x)$ be its $N$-finite sum:

$$
g_{N}(t, x)=\sum_{n=0}^{N} g_{n}(t) \psi_{n}(x) .
$$

Then it follows that

$$
\left\{\begin{array}{l}
g_{N}(t, x) \longrightarrow g(t, x) \text { in } L^{2}\left(\left[T^{*}, T\right] \times \boldsymbol{R}^{+}\right) \\
\partial_{t} g_{N}(t, x) \longrightarrow \partial_{t} g(t, x) \text { in } L^{2}\left(\left[T^{*}, T\right] \times \boldsymbol{R}^{+}\right) \text {as } N \rightarrow \infty
\end{array}\right.
$$

and $g_{N}(t, x) \in L^{2}\left(\left[T^{*}, T\right], \Phi_{3}(T)\right)$.

Now we rewrite (4.11.j) as follows:

$$
\left\{\begin{array}{l}
i \partial_{t} u=(-1 / 2) \partial_{x}^{2} u+x t^{\kappa} u+g \quad \text { in }\left(T^{*}, T\right) \times \boldsymbol{R}^{+} \\
\left.u\right|_{x=0}=0,\left.\quad u\right|_{t=T}=u_{m}(x) \\
u_{m}(x) \in \mathcal{S}\left(\overline{\boldsymbol{R}}^{+}\right) \\
\text {and } \\
g \in C^{0}\left(\left[T^{*}, T\right], L^{2}\left(\boldsymbol{R}^{+}\right)\right) \text {satisfies }(4.12) \text { for some } \\
\left\{g_{N}(t, x)\right\} \subset L^{2}\left(\left[T^{*}, T\right], \Phi_{3}(T)\right) .
\end{array}\right.
$$

If we can find the solution $u(t, x)$ to (4.13), the lemma is proved.

By Lemma 4.1, there is a solution $u_{N}(t, x) \in L^{2}\left(\left[T^{*}, T\right], \Phi_{3}(T)\right)$ to

$$
\left[\begin{array}{l}
i \partial_{t} u_{N}=(-1 / 2) \partial_{x}^{2} u_{N}+x t^{\kappa} u_{N}+g_{N} \\
\left.u_{N}\right|_{x=0}=0,\left.\quad u_{N}\right|_{t=T}=u_{m}(x) \quad \text { for each } N(N=0,1,2, \cdots) .
\end{array}\right.
$$

From the energy inequality (cf. Lemma 4.3), we have

$$
\begin{aligned}
& \left\|u_{N}(t, \cdot)\right\|+t^{x}\left\|x u_{N}(t, \cdot)\right\|+\left\|\partial_{x}^{2} u_{N}(t, \cdot)\right\| \\
& \leqq \\
& \quad C\left[\left\|u_{N}(T, \cdot)\right\|+(T / t)^{\kappa}\left\|\partial_{x}^{2} u_{N}(T, \cdot)\right\|+\left(T^{2} / t\right)^{\kappa}\left\|x u_{N}(T, \cdot)\right\|\right. \\
& \quad+(T / t)^{\kappa}\left\|g_{N}(T, \cdot)\right\|+\left\|g_{N}(t, \cdot)\right\|+\int_{t}^{T}\left\{\left\|g_{N}(s, \cdot)\right\|\right. \\
& \left.\left.\quad+\kappa(1 / s)(s / t)^{\kappa}\left\|g_{N}(s, \cdot)\right\|+(s / t)^{\kappa}\left\|g_{N}^{\prime}(s, \cdot)\right\|\right\} d s\right] .
\end{aligned}
$$

On the other hand, since 


$$
\left[\begin{array}{l}
i \partial_{t}\left(u_{N}-u_{M}\right)=(-1 / 2) \partial_{x}^{2}\left(u_{N}-u_{M}\right)+x t^{\kappa}\left(u_{N}-u_{M}\right)+g_{N}-g_{M} \\
\left.\left(u_{N}-u_{M}\right)\right|_{x=0}=0,\left.\quad\left(u_{N}-u_{M}\right)\right|_{t=T}=0
\end{array}\right.
$$

we have

$$
\begin{aligned}
& \left\|u_{N}(t, \cdot)-u_{M}(t, \cdot)\right\|+t^{\kappa}\left\|x u_{N}(t, \cdot)-x u_{M}(t, \cdot)\right\|+\left\|\partial_{x}^{2} u_{N}(t, \cdot)-\partial_{x}^{2} u_{M}(t, \cdot)\right\| \\
& \leqq \\
& \quad C\left\{(T / t)^{\kappa}\left\|g_{N}(T, \cdot)-g_{M}(T, \cdot)\right\|+\left\|g_{N}(t, \cdot)-g_{M}(t, \cdot)\right\|\right. \\
& \quad+\int_{t}^{T}\left(\left\|g_{N}(s, \cdot)-g_{M}(s, \cdot)\right\|+\kappa(1 / s)(s / t)^{\kappa}\left\|g_{N}(s, \cdot)-g_{M}(s, \cdot)\right\|\right. \\
& \left.\left.\quad+(s / t)^{x}\left\|g_{N}^{\prime}(s, \cdot)-g_{M}^{\prime}(s, \cdot)\right\|\right) d s\right\} .
\end{aligned}
$$

Hence applying Schwarz's inequality, we have

$$
\begin{aligned}
& \int_{T *}^{T}\left\|u_{N}(t, \cdot)-u_{M}(t, \cdot)\right\|^{2} d t+\left(T^{*}\right)^{2 n} \int_{T *}^{T}\left\|x u_{N}(t, \cdot)-x u_{M}(t, \cdot)\right\|^{2} d t \\
& +\int_{T *}^{T}\left\|\partial_{x}^{2} u_{N}(t, \cdot)-\partial_{x}^{2} u_{M}(t, \cdot)\right\|^{2} d t \\
& \leqq C\left[\left(T / T^{*}\right)^{2 \kappa}\left(T-T^{*}\right)\left\|g_{N}(T, \cdot)-g_{M}(T, \cdot)\right\|^{2}+\int_{T *}^{T}\left\|g_{N}(t, \cdot)-g_{M}(t, \cdot)\right\|^{2} d t\right. \\
& +(1 / 2)\left(T-T^{*}\right)^{2}\left\{\int_{T *}^{T}\left\|g_{N}(\tau, \cdot)-g_{M}(\tau, \cdot)\right\|^{2} d \tau\right. \\
& +\kappa^{2}\left(1 / T^{2}\right)\left(T / T^{*}\right)^{2 \kappa} \int_{T *}^{T}\left\|g_{N}(\tau, \cdot)-g_{M}(\tau, \cdot)\right\|^{2} d \tau \\
& \left.\left.+\left(T / T^{*}\right)^{2 \kappa} \int_{T *}^{T}\left\|g_{N}^{\prime}(\tau, \cdot)-g_{M}^{\prime}(\tau, \cdot)\right\|^{2} d \tau\right\}\right] .
\end{aligned}
$$

Then it is clear that the limit

$$
u(t, x)=\lim _{N \rightarrow \infty} u_{N}(t, x) \in L^{2}\left(\left[T^{*}, T\right], \Phi_{2}(T)\right)
$$

is the unique solution to (4.13), and Lemma 4.6 is proved.

Q.E.D.

Lemma 4.7. There exists a solution $v(t, x) \in L^{2}\left(\left[T^{*}, T\right], \Phi_{2}(T)\right)$ to (4.10).

Proof. By (4.14) and Minkowski's inequality, we have

$$
\begin{aligned}
& \int_{t}^{T}\left\|u_{N}(\tau, \cdot)\right\|^{2} d \tau+\left(T^{*}\right)^{2 \kappa} \int_{t}^{T}\left\|x u_{N}(\tau, \cdot)\right\|^{2} d \tau+\int_{t}^{T}\left\|\partial_{x}^{2} u_{N}(\tau, \cdot)\right\|^{2} d \tau \\
& \leqq C\left[(T-t)\left\|u_{N}(T, \cdot)\right\|^{2}+\left(T / T^{*}\right)^{2 \kappa}(T-t)\left\|\partial_{x}^{2} u_{N}(T, \cdot)\right\|^{2}\right. \\
& \quad+\left(T^{2} / T^{*}\right)^{2 \kappa}(T-t)\left\|x u_{N}(T, \cdot)\right\|^{2}+\left(T / T^{*}\right)^{2 \kappa}(T-t)\left\|g_{N}(T, \cdot)\right\|^{2}
\end{aligned}
$$




$$
\begin{aligned}
& +\int_{t}^{T}\left\|g_{N}(\tau, \cdot)\right\|^{2} d \tau+(1 / 2)(T-t)^{2}\left\{\int_{t}^{T}\left\|g_{N}(\tau, \cdot)\right\|^{2} d \tau\right. \\
& \left.+\kappa^{2}(1 / T)^{2}\left(T / T^{*}\right)^{2 \kappa} \int_{t}^{T}\left\|g_{N}(\tau, \cdot)\right\|^{2} d \tau\right\} \\
& \left\{\begin{array}{l}
\left.+(1 / 2)\left(T / T^{*}\right)^{2 \kappa}(T-t)^{2} \int_{t}^{T}\left\|g_{N}^{\prime}(\tau, \cdot)\right\|^{2} d \tau\right] \\
\left.+\left(T / T^{*}\right)^{2 \kappa}(T-t) \int_{t}^{T} \int_{s}^{T}\left\|g_{N}^{\prime}(\tau, \cdot)\right\|^{2} d \tau d s\right] .
\end{array}\right.
\end{aligned}
$$

Letting $N \rightarrow \infty$, and applying Lebesgue's dominated convergence theorem, this implies

$$
\begin{aligned}
& \int_{t}^{T}\|u(\tau, \cdot)\|^{2} d \tau+\left(T^{*}\right)^{2 \kappa} \int_{t}^{T}\|x u(\tau, \cdot)\|^{2} d \tau+\int_{t}^{T}\left\|\partial_{x}^{2} u(\tau, \cdot)\right\|^{2} d \tau \\
& \leqq C\left[(T-t)\|u(T, \cdot)\|^{2}+\left(T / T^{*}\right)^{2 \kappa}(T-t)\left\|\partial_{x}^{2} u(T, \cdot)\right\|^{2}\right. \\
& +\left(T^{2} / T^{*}\right)^{2 \kappa}(T-t)\|x u(T, \cdot)\|^{2}+\left(T / T^{*}\right)^{2 \kappa}(T-t)\|g(T, \cdot)\|^{2} \\
& +\int_{t}^{T}\|g(\tau, \cdot)\|^{2} d \tau+(1 / 2)(T-t)^{2}\left\{\int_{t}^{T}\|g(\tau, \cdot)\|^{2} d \tau\right. \\
& \left.+\kappa^{2}(1 / T)^{2}\left(T / T^{*}\right)^{2 \kappa} \int_{t}^{T}\|g(\tau, \cdot)\|^{2} d \tau\right\} \\
& \left\{\begin{array}{l}
\left.+(1 / 2)\left(T / T^{*}\right)^{2 \kappa}(T-t)^{2} \int_{t}^{T}\left\|g^{\prime}(\tau, \cdot)\right\|^{2} d \tau\right] \\
\left.+\left(T / T^{*}\right)^{2 \kappa}(T-t) \int_{t}^{T} \int_{s}^{T}\left\|g^{\prime}(\tau, \cdot)\right\|^{2} d \tau d s\right] .
\end{array}\right.
\end{aligned}
$$

Therefore, we have proved that (4.13) admits a unique solution $u(t, x) \in$ $L^{2}\left(\left[T^{*}, T\right], \Phi_{2}(T)\right)$ with the energy inequality (4.15) whenever $g(t, x) \in C^{0}\left(\left[T^{*}, T\right]\right.$, $\left.L^{2}\left(\boldsymbol{R}^{+}\right)\right)$and $\partial_{t} g(t, x) \in L^{2}\left(\left[T^{*}, T\right], L^{2}\left(\boldsymbol{R}^{+}\right)\right)$exists for almost everywhere $t \in$ $\left[T^{*}, T\right]$. From (4.11.j),

$$
\begin{aligned}
& i \partial_{t}\left(v_{j+1}-v_{\jmath}\right)+(1 / 2) \partial_{x}^{2}\left(v_{\jmath+1}-v_{\jmath}\right)-x t^{\kappa}\left(v_{j+1}-v_{\jmath}\right) \\
& \quad=\sum_{l=1}^{r}\left(\begin{array}{c}
m \\
l
\end{array}\right) x\left(\partial_{t}^{l} t^{\kappa}\right) \int_{t}^{T} \frac{(t-s)^{l-1}}{(l-1) !}\left(v_{j}-v_{j-1}\right)(s, x) d s \\
& \left(v_{j+1}-v_{j}\right)(T, x)=0, \quad\left(v_{j+1}-v_{j}\right)(t, 0)=0, \quad v_{0}(t, x) \equiv 0 .
\end{aligned}
$$

Applying (4.15) to the above,

$$
\begin{aligned}
& \int_{t}^{T}\left\|\left(v_{j+1}-v_{j}\right)(\tau, \cdot)\right\|^{2} d \tau+\left(T^{*}\right)^{2 \kappa} \int_{t}^{T}\left\|x\left(v_{j+1}-v_{\jmath}\right)(\tau, \cdot)\right\|^{2} d \tau \\
& \quad+\int_{t}^{T}\left\|\partial_{x}^{2}\left(v_{j+1}-v_{j}\right)(\tau, \cdot)\right\|^{2} d \tau \\
& \leqq \\
& \quad C\left(T, T^{*}, m, \kappa\right) \int_{t}^{T} \int_{\tau}^{T}\left\|x\left(v_{j}-v_{j-1}\right)(s, \cdot)\right\|^{2} d s d \tau .
\end{aligned}
$$


Here we have used the estimate:

$$
\begin{aligned}
& \int_{t}^{T}\left\|\sum_{l=1}^{r}\left(\begin{array}{c}
m \\
l
\end{array}\right)\left(\partial_{\tau}^{l} \tau^{k}\right) \int_{\tau}^{T} \frac{(\tau-s)^{l-1}}{(l-1) !} x\left(v_{j}-v_{j-1}\right)(s, \cdot) d s\right\|^{2} d \tau \\
& \quad \leqq \int_{t}^{T}\left\{\int_{\tau}^{T} \sum_{l=1}^{r}\left(\begin{array}{c}
m \\
l
\end{array}\right)\left(\partial_{\tau}^{l} \tau^{\kappa}\right) \frac{(\tau-s)^{l-1}}{(l-1) !}\left\|x\left(v_{j}-v_{j-1}\right)(s, \cdot)\right\| d s\right\}^{2} d \tau \\
& \quad \leqq \int_{t}^{T}\left\{\int_{\tau}^{T}\left|\sum_{l=1}^{r}\left(\begin{array}{c}
m \\
l
\end{array}\right)\left(\partial_{\tau}^{l} \tau^{\kappa}\right) \frac{(\tau-s)^{l-1}}{(l-1) !}\right|^{2} d s\right\}\left\{\int_{\tau}^{T}\left\|x\left(v_{j}-v_{j-1}\right)(s, \cdot)\right\|^{2} d s\right\} d \tau .
\end{aligned}
$$

Now by Lemma 4.1 ,

$$
\int_{\tau}^{T}\left\|x\left(v_{1}-v_{0}\right)(s, \cdot)\right\|^{2} d s \leqq M
$$

Hence by putting

$$
\begin{aligned}
w_{\jmath}(t)= & \int_{t}^{T}\left\|\left(v_{j+1}-v_{j}\right)(\tau, \cdot)\right\|^{2} d \tau+\left(T^{*}\right)^{2 \kappa} \int_{t}^{T}\left\|x\left(v_{j+1}-v_{j}\right)(\tau, \cdot)\right\|^{2} d \tau \\
& +\int_{t}^{T}\left\|\partial_{x}^{2}\left(v_{j+1}-v_{j}\right)(\tau, \cdot)\right\|^{2} d \tau
\end{aligned}
$$

we have

$$
w_{1}(t) \leqq C \int_{t}^{T} M d \tau=C M(T-t), \quad \text { and } \quad w_{j+1}(t) \leqq C \int_{t}^{T} w_{j}(\tau) d \tau
$$

So

$$
w_{j}(t) \leqq M \frac{\{C(T-t)\}^{j}}{j !} \leqq M \frac{\left\{C\left(T-T^{*}\right)\right\}^{j}}{j !}
$$

which tends to zero as $j \rightarrow \infty$. Therefore $\left\{v_{j}(t, x)\right\}_{j=0}^{\infty}$ is a Cauchy sequence in $L^{2}\left(\left[T^{*}, T\right], \Phi_{2}(T)\right)$, and there exist a solution $v(t, x) \in L^{2}\left(\left[T^{*}, T\right], \Phi_{2}(T)\right)$ to (4.10) such that

$$
v_{j}(t, x) \longrightarrow v(t, x) \quad \text { as } j \rightarrow \infty .
$$

This completes the proof of Lemma 4.7.

Q.E.D.

From the above arguments, we have constructed $\tilde{u}(t, x)$ of the form (4.8) satisfying (3.6). This shows that

and

$$
\tilde{u}(t, x) \in C^{m}\left(\left[T^{*}, T\right], L^{2}\left(\boldsymbol{R}^{+}\right)\right),
$$

$$
\partial_{x}^{2} \tilde{u}(t, x), x \tilde{u}(t, x) \in C^{m-1}\left(\left[T^{*}, T\right], L^{2}\left(\boldsymbol{R}^{+}\right)\right) .
$$

Also, by Corollary $4.4, \tilde{u}(t, x)=u(t, x)$ where $u(t, x)$ is the solution to (3.6). Summing up, we have proved the following result.

Proposition 4.8. Let $u(t, x)$ be the solution to (3.6). Then

and

$$
u(t, x) \in C^{m}\left(\left[T^{*}, T\right], L^{2}\left(\boldsymbol{R}^{+}\right)\right),
$$


that is

$$
\partial_{x}^{2} u(t, x), \quad x u(t, x) \in C^{m-1}\left(\left[T^{*}, T\right], L^{2}\left(\boldsymbol{R}^{+}\right)\right),
$$

$$
u(t, x) \in C^{m-1}\left(\left[T^{*}, T\right], \Phi_{2}(T)\right) .
$$

Next, by using this result, we show that if $t \in\left[T^{*}, T\right], u(t, x)$ has higher order derivatives with respect to $x$, and is rapidly decreasing as $x \rightarrow+\infty$.

Before going further we introduce some notation which will be frequently used in the subsequent arguments.

Notation. We define the $\Phi_{n}$-norm $\|u(t, \cdot)\|_{\Phi_{n}}(n \geqq 0)$ of $u(t, \cdot)$ by $\|u(t, \cdot)\|_{\Phi_{n}}$ $=\sum_{p=0}^{n}\left\|x^{p / 2} \partial_{x}^{n-p} u(t, \cdot)\right\|$, and call the set $\left\{x^{p / 2} \partial_{x}^{n-p} u(t, x), 0 \leqq p \leqq n\right\} \Phi_{n}$-class of $u(t, x)$.

Lemma 4.9. Let $u(t, x) \in C^{m}\left(\left[T^{*}, T^{-}\right], L^{2}\left(\boldsymbol{R}^{+}\right)\right) \cap C^{m-1}\left(\left[T^{*}, T\right], \Phi_{2}(T)\right)$ be the solution to (3.6). Then $x^{p / 2} \partial_{x}^{2 m-p} u(t, x) \in L^{\infty}\left(\left[T^{*}, T\right], L^{2}\left(\boldsymbol{R}^{+}\right)\right), 0 \leqq p \leqq 2 m$.

Proof. We will show that the $\Phi_{2 n}$-norm $(n \leqq m)$ of $u(t, \cdot)$ is in $L^{\infty}\left(\left[T^{*}, T\right]\right)$. The proof is done by induction on $n$. By Proposition 4.8 , this is true for the case $n=1$. Suppose that the $\Phi_{2 k}$-norm $(k \leqq n)$ is in $L^{\infty}\left(\left[T^{*}, T\right]\right)$. If we integrate $\int_{0}^{\infty} x \partial_{t}^{n} u \cdot x \partial_{t}^{n-1} u d x<\infty\left(t \in\left[T^{*}, T\right]\right)$ by parts using (3.6), $\left\|x^{(2+p) / 2} \partial_{x}^{2 n-1-p} u\right\|^{2}$ $0 \leqq p \leqq 2 n-1$ appear as its positive parts, and the other terms are bounded by the $\Phi_{2 k}$-norms $(k \leqq n)$. So we have $x^{(2+p) / 2} \partial_{x}^{2 n-1-p} u \in L^{\infty}\left(\left[T^{*}, T\right], L^{2}\left(\boldsymbol{R}^{+}\right)\right) 0 \leqq p$ $\leqq 2 n-1$. Furthermore, $\partial_{t}^{n} \partial_{x} u \in L^{\infty}\left(\left[T^{*}, T\right], L^{2}\left(\boldsymbol{R}^{+}\right)\right)$implies $\partial_{x}^{2 n+1} u \in L^{\infty}\left(\left[T^{*}, T\right]\right.$, $\left.L^{2}\left(\boldsymbol{R}^{+}\right)\right)$, and from $\int_{0}^{\infty} \partial_{x}^{2 n+1} u \cdot x \overline{\partial_{x}^{2 n-1} u} d x<\infty\left(t \in\left[T^{*}, T\right]\right)$, we have $x^{1 / 2} \partial_{x}^{2 n} u \in$ $L^{\infty}\left(\left[T^{*}, T\right], L^{2}\left(\boldsymbol{R}^{+}\right)\right)$. Thus the $\Phi_{2 n+1}$-norm of $u(t, \cdot)$ is in $L^{\infty}\left(\left[T^{*}, T\right]\right)$. In the same way, integrating $\int_{0}^{\infty} x \partial_{t}^{n} u x \overline{\partial_{t}^{n} u} d x<\infty\left(t \in\left[T^{*}, T\right]\right)$ by parts, it follows

$$
x^{(2+p) / 2} \partial_{x}^{2 n-p} u \in L^{\infty}\left(\left[T^{*}, T\right], L^{2}\left(\boldsymbol{R}^{+}\right)\right) \quad 0 \leqq p \leqq 2 n,
$$

and $\partial_{t}^{n} \partial_{x}^{2} u \in L^{\infty}\left(\left[T^{*}, T\right], L^{2}\left(\boldsymbol{R}^{+}\right)\right)$implies $\partial_{x}^{2 n+2} u \in L^{\infty}\left(\left[T^{*}, T\right], L^{2}\left(\boldsymbol{R}^{+}\right)\right)$. Moreover, from $\int_{0}^{\infty} \partial_{x}^{2 n+2} u \cdot x \overline{\partial_{x}^{2 n} u} d x<\infty\left(t \in\left[T^{*}, T\right]\right)$, it follows

$$
x^{1 / 2} \partial_{x}^{2 n+1} u \in L^{\infty}\left(\left[T^{*}, T\right], L^{1}\left(\boldsymbol{R}^{+}\right)\right) .
$$

Thus the $\Phi_{2 n+2}$-norm of $u(t, \cdot)$ is in $L^{\infty}\left(\left[T^{*}, T\right]\right)$.

Q.E.D.

Finally, we show Lemma 4.9 still holds for $T^{*}=0$, and also prove the existence of a function $\rho(N)$ satisfying (3.1), (3.2) and (3.3).

Lemma 4.10. Suppose that

$$
\operatorname{Sup}_{x \geq 0}\left|t^{k} x^{l} \partial_{t}^{m} \partial_{x}^{n} h(t, x)\right| \longrightarrow 0 \quad(t \rightarrow+\infty)
$$


for any nonnegative integer $k, l, m, n$ with

$$
k+l+m+n \leqq \kappa\left(N^{2}+2 N\right)+3 N+2
$$

and let $u(t, x)$ be the solution to (3.5). Then for each nonnegative integer $k, l$, $m$, $n$ such that $2 k+2 l+2 m+n \leqq 2 N\left\|t^{k} x^{l} \partial_{t}^{m} \partial_{x}^{n} u(t, \cdot)\right\|$ is bounded for all $t(0 \leqq t<\infty)$.

Proof. First we prove that each $\Phi_{m}$-norm of $u(t, x)\left(m=0,1,2, \cdots, 2 N^{\top}\right)$ is bounded as $t \rightarrow+0$. We prove this by induction on $m$.

Since (4.5) implies $\|u(t, \cdot)\| \leqq\|h(T, \cdot)\|$, this is true for $m=0$. Now assume that $\Phi_{m}$-norm $(m \leqq 2 n)$ is bounded as $t \rightarrow+0$. From (3.5) we can easily derive for $n=0,1,2, \cdots, N-1$

$$
\left\{\begin{array}{l}
i \partial_{t}\left(x^{n} \partial_{x} u\right)=(-1 / 2) \partial_{x}^{2}\left(x^{n} \partial_{x} u\right)+x t^{\kappa}\left(x^{n} \partial_{x} u\right)+\cdots, \\
i \partial_{t}\left(x^{n+1} u\right)=(-1 / 2) \partial_{x}^{2}\left(x^{n+1} u\right)+x t^{\kappa}\left(x^{n+1} u\right)+x^{n} \partial_{x} u+\cdots, \\
i \partial_{t}\left(x^{n-k-1} \partial_{x} \partial_{t}^{1+k} u\right)=(-1 / 2) \partial_{x}^{2}\left(x^{n-k-1} \partial_{x} \partial_{t}^{1+k} u\right) \\
\quad+x t^{\kappa}\left(x^{n-k-1} \partial_{x} \partial_{t}^{1+k} u\right)+c \kappa t^{\kappa-1} x^{n-k} \partial_{x} \partial_{t}^{k} u+\cdots \quad \text { for } k=0,1, \cdots, n-1, \\
i \partial_{t}\left(x^{n-k} \partial_{t}^{1+k} u\right)=(-1 / 2) \partial_{x}^{2}\left(x^{n-k} \partial_{t}^{1+k} u\right)+x t^{k}\left(x^{n-k} \partial_{t}^{1+k} u\right) \\
\quad+c \kappa t^{k-1} x^{n+1-k} \partial_{t}^{k} u+c x^{n-1-k} \partial_{x} \partial_{t}^{k+1} u+\cdots \quad \text { for } k=0,1,2, \cdots, n .
\end{array}\right.
$$

Here $\cdots \cdots$ means the terms of the functions in $\Phi_{m}(m \leqq 2 n)$ class. Using these equations and $L^{2}$ estimate (4.5) repeatedly, we find that the $\Phi_{m}$-norm $(m \leqq 2 n+2)$ of $u(t, x)$ is bounded as $t \rightarrow+0$. This completes the induction.

Next, by roughly estimating the number of $t$ included in each term of (4.16), each $\left\|x^{l} \partial_{t}^{m} \partial_{x}^{n} u(t, \cdot)\right\|(2 l+2 m+n \leqq 2 N)$ is estimated from above by $\left\|T^{k} x^{l} \partial_{T}{ }^{m} \partial_{x}{ }^{n} h(T, \cdot)\right\|\left(k \leqq \kappa\left(N^{2}+2 N\right), 2 l+2 m+n \leqq 2 N\right)$. Moreover from

$$
i \partial_{t}\left(t^{n} u\right)+(1 / 2) \partial_{x}^{2}\left(t^{n} u\right)-x t^{\kappa}\left(t^{n} u\right)=i n t^{n-1} u \quad(n=1,2, \cdots) .
$$

$\left\|t^{k} u(t, \cdot)\right\|$ is estimated from above by $\left\|T^{k} h(T, \cdot)\right\|$. Summing up, each $\left\|t^{k} x^{l} \partial_{t}{ }^{m} \partial_{x}{ }^{n} u(t, \cdot)\right\|(2 k+2 l+2 m+n \leqq 2 N)$ is estimated by $\left\|T^{k} x^{l} \partial_{t}{ }^{m} \partial_{x}{ }^{n} h(T, \cdot)\right\|$ $\left(k+l+m+n \leqq \kappa\left(N^{2}+2 N\right)+3 N\right)$. This completes the proof. Q.E. D.

Finally we remark about the proof of the existence of $\rho(N)$. First note that

$$
\left\|T^{k} x^{l} \partial_{t}{ }^{m} \partial_{x}{ }^{n} h(T, \cdot)\right\| \quad\left(k+l+m+n \leqq \kappa\left(N^{2}+2 N\right)+3 N\right)
$$

is integrable with respect to $T$ on $[0, \infty)$ if

$$
\operatorname{Sup}_{T \geqq 0}\left\|T^{k} x^{l} \partial_{t}{ }^{m} \partial_{x}{ }^{n} h(T, \cdot)\right\|<\infty \quad\left(k+l+m+n \leqq \kappa\left(N^{2}+2 N\right)+3 N+2\right) .
$$

Then using Duhamel's principle and the stimate stated in the proof of Lemma 4.10, we have

$$
\left\|t^{k} x^{l} \partial_{t}{ }^{m} \partial_{x}{ }^{n} w(t, \cdot)\right\|<\infty \quad(2 k+2 l+2 m+n \leqq 2 N, t \geqq 0) .
$$


This shows the existence of a function $\rho(N)$ such that

$$
\rho(N) \longrightarrow \infty \quad \text { as } N \rightarrow \infty \text {. }
$$

Thus we have proved Theorem 2.3.

\section{References}

[1] Babich, V.M. and Kirpichnikova, N.Y., The Boundary-Layer Method in Diffraction Problems, Springer, 1979.

[2] Babich, V.M. and Smyshlyaev, V.P., The Scattering Problem for the Schrödinger Equation with a Potential Linear in Time and in Space. 2. Correctness, Smoothness, Behavior of the Solution at Infinity, J. Soviet. Math., 38 (1987), 1562-1576.

[3] Mizohata, S., The Theory of Partial Differential Equations, Cambridge Univ. Press, 1973.

[4] Popov, M.M., Problem of Whispering Gallery Waves in a Neighborhood of a Simple Zero of the Effective Curvature of the Boundary, J. Soviet. Math., 11 (1979), 791797.

[5] Titchmarsh, E. C., Eigenfunction Expansions, Part 1, second edition, Oxford, 1962. 
